\title{
Controlled School Choice with Soft Bounds and Overlapping Types
}

\author{
Ryoji Kurata \\ Naoto Hamada \\ Kyushu University
}

Atsushi Iwasaki

University of Electro-Communications

1-5-1 Chofugaoka, Chofu

Tokyo, 182-8585 Japan

\section{Makoto Yokoo}

Kyushu University

744 Motooka, Nishi-ku

Fukuoka, 819-0395 Japan

\author{
KURATA@AGENT.INF.KYUSHU-U.AC.JP \\ NHAMADA@AGENT.INF.KYUSHU-U.AC.JP
}

IWASAKI@IS.UEC.AC.JP

YOKOO@INF.KYUSHU-U.AC.JP

\begin{abstract}
School choice programs are implemented to give students/parents an opportunity to choose the public school the students attend. Controlled school choice programs need to provide choices for students/parents while maintaining distributional constraints on the composition of students, typically in terms of socioeconomic status. Previous works show that setting soft-bounds, which flexibly change the priorities of students based on their types, is more appropriate than setting hard-bounds, which strictly limit the number of accepted students for each type. We consider a case where soft-bounds are imposed and one student can belong to multiple types, e.g., "financially-distressed" and "minority" types. We first show that when we apply a model that is a straightforward extension of an existing model for disjoint types, there is a chance that no stable matching exists. Thus we propose an alternative model and an alternative stability definition, where a school has reserved seats for each type. We show that a stable matching is guaranteed to exist in this model and develop a mechanism called Deferred Acceptance for Overlapping Types (DA-OT). The DA-OT mechanism is strategy-proof and obtains the student-optimal matching within all stable matchings. Furthermore, we introduce an extended model that can handle both type-specific ceilings and floors and propose a extended mechanism DA-OT* to handle the extended model. Computer simulation results illustrate that DA-OT outperforms an artificial cap mechanism where we set a hard-bound for each type in each school. DA-OT* can achieve stability in the extended model without sacrificing students' welfare.
\end{abstract}

\section{Introduction}

The theory of two-sided matching has been developed and applied to various markets in practice. $^{1}$ Also, there is a growing interest for this topic among AI and multiagent sys-

1. See the book by Roth and Sotomayor (1990) or Manlove (2013) for a comprehensive survey of many results in this literature. 
tems researchers, e.g., handling optimization problems in kidney exchange (Awasthi \& Sandholm, 2009), compactly representing preferences (Pini, Rossi, \& Venable, 2014), handling partial preferences (Drummond \& Boutilier, 2013a; Rastegari, Condon, Immorlica, \& Leyton-Brown, 2013), and so on.

In many application domains, various distributional constraints are often imposed on an outcome, e.g., regional maximum quotas are imposed on hospitals in urban areas so that more doctors are allocated to rural areas (Kamada \& Kojima, 2015), or minimum quotas are imposed when school districts require that at least a certain number of students is allocated to each school so that the school can operate properly (Biró, Fleiner, Irving, \& Manlove, 2010; Fragiadakis, Iwasaki, Troyan, Ueda, \& Yokoo, 2016; Goto, Hashimoto, Iwasaki, Kawasaki, Ueda, Yasuda, \& Yokoo, 2014).

This paper deals with yet another type of distributional constraint, i.e., diversity constraints in school choice programs. School choice programs are implemented to give students/parents an opportunity to choose the public school the students attend. However, a school is required to balance its composition of students, typically in terms of socioeconomic status. Controlled school choice programs need to provide choices for students/parents while maintaining distributional constraints.

A seminal work by Abdulkadiroğlu and Sönmez (2003) proposes using the Deferred Acceptance (DA) mechanism in school choice programs. Kojima (2012) considers a model with two types of students, i.e., minority and majority, and shows that setting hard-bounds for the number of majority students may hurt the minority students. To overcome this shortcoming, Hafalir, Yenmez, and Yildirim (2013) propose soft-bounds for the number of minority students, i.e., schools give higher priority to minority students up to a certain number. Ehlers, Hafalir, Yenmez, and Yildirim (2014) generalize the model by Hafalir et al. (2013) to cases where the number of student types can exceed two. Ehlers et al. show several impossibility results when these minimum quotas are hard constraints and obtain positive results when they are soft-bounds.

Our work is based on the work of Ehlers et al. (2014) and examines a more general case where one student can belong to multiple types, e.g., to both "financially-distressed" and "minority" types. Although we can assume all types are disjoint by enumerating all the combinations of types and consider each combination a different type (e.g., financially-distressed minority, financially-distressed majority, financially-sound minority, and financially-sound majority), setting an appropriate quota for each finely divided type is difficult. Furthermore, when the number of types increases, considering all the combinations and setting appropriate goals for each type combination becomes impractical. As far as the authors are aware, no previous work has considered a model where a student can belong to multiple types with soft-bounds.

In this paper, we first show a model that is a straightforward extension of the one used by Ehlers et al. (2014). It turns out that we cannot guarantee the existence of a stable matching in this model. Thus we propose an alternative model and an alternative stability definition, where a school has reserved seats for each type, and stability is defined based on the number of students who are assigned to the reserved seats. We show that a stable matching is guaranteed to exist and develop a mechanism called Deferred Acceptance for Overlapping Types (DA-OT) that obtains a stable matching. DA-OT mechanism is strategy-proof and obtains the student-optimal matching within all stable matchings. 
Furthermore, we introduce an extended model that can handle both type-specific ceilings and floors, and show how to modify DA-OT to handle the extended model. We also show computer simulation results, which illustrate that DA-OT outperforms an artificial cap mechanism, where the number of reserved seats of each type is fixed.

The rest of this paper is organized as follows. First, in the rest of this section, we additionally explore some literature in matching with constraints. Second, we introduce a basic model that is a straightforward extension of Ehlers et al. (2014) and show that there is a chance that no stable matching exists in Section 2.1. Then, in Section 2.2, we introduce a new basic model and a new stability definition, where a school has reserved seats for each type, and stability is defined based on the number of students who are assigned to the reserved seats. Third, we introduce our new mechanism that can work on the new basic model (Section 3) and show its properties (Section 4). Furthermore, we introduce an extended model that applies two parameters, i.e., a floor and a ceiling, that specify a non-binding target range (Section 5). Finally, we describe computer simulation results to show the performance of our new mechanisms (Section 6).

\subsection{Related Literature}

In recent years, matching problems with diversity constraints or affirmative actions, have been broadly studied. In public school choice programs, for example, students/parents can choose a school to attend, while some (or all) schools may be required to satisfy a certain balance of the composition of students, typically in terms of socioeconomic status. Since Abdulkadiroğlu and Sönmez (2003) proposed using DA mechanism in school choice programs, besides the papers we have already referred to, many studies have addressed how we should modify it to control the balance of the composition of students.

Abdulkadiroğlu (2005), and Ergin and Sönmez (2006) employ only type-specific ceilings as hard-bounds. By lowering ceilings for a majority type, they try to fill seats or slots for a minority type. Note that such mechanisms, which we call artificial cap deferred acceptance mechanisms will be used as a benchmark in Section 6. In contrast, our mechanisms simultaneously employ both type-specific ceilings and floors as soft-bounds.

Westkamp (2013) examines another type of mechanisms which sequentially consider students of different types in the context of German university admissions inspired by the empirical analysis by Braun, Dwenger, Kübler, and Westkamp (2014). Kominers and Sönmez (2015) consider a model where each seat/slot of a school has a different priority ranking for students. This model can represent certain types of affirmative action and can be considered a generalization of the model used by Hafalir et al. (2013). Echenique and Yenmez (2015) provide a characterization of strategy-proof mechanisms, which is similar to the ones by Westkamp (2013) and Kominers and Sönmez (2015). Dur, Kominers, Pathak, and Sönmez (2013) study how to control school priorities over sets of students and how to handle diversity constraints in the context of Boston school choice. Budish, Che, Kojima, and Milgrom (2010) develop a randomized mechanism which achieves a feasible assignment in expectation that satisfies type-specific ceilings. These studies assume that each student belongs to a single type. Our paper is substantially different from them because we assume that each student can belong to multiple types, i.e., overlapping types. 
As mentioned earlier, various distributional constraints are often imposed on an outcome in many application domains. Kamada and Kojima (2015) and Goto, Iwasaki, Kawasaki, Yasuda, and Yokoo (2014) consider regional maximum quotas. Biró et al. (2010), Sönmez and Switzer (2013), Monte and Tumennasan (2013), Goto et al. (2014), and Fragiadakis et al. (2016) discuss how to handle minimum quotas. In the literature of computer science, the complexity of checking the existence of a stable matching has also been discussed when various distributional constraints are imposed (Huang, 2010; Fleiner \& Kamiyama, 2016; Hamada, Iwama, \& Miyazaki, 2016; Kamiyama, 2013). One fundamental difference between these works and ours is that they consider distributional constraints as hard constraints, which must be completely satisfied. Our work (as well as most of the works on school choice programs mentioned earlier) assumes that constraints are soft-bounds, reflecting the negative results regarding hard-bounds pointed out by Kojima (2012) and Ehlers et al. (2014).

We use the framework of matching with contracts due to Hatfield and Milgrom (2005). ${ }^{2}$ They show that if the choice function of every school satisfies several conditions, a mechanism called a generalized Deferred Acceptance (DA) mechanism finds a "stable" allocation, and it is strategy-proof for students.

There are several recent works related to a general framework for handling various distributional constraints. Kojima, Tamura, and Yokoo (2014) propose a general framework that can handle a more general class of constraints (represented by matroidal constraints and an $\mathrm{M}^{\natural}$-concave function). Their framework provides an alternative method to the one developed by Hatfield and Milgrom (2005). It seems possible to apply their framework in our setting. However, we apply a more familiar, standard framework proposed by Hatfield and Milgrom. It must be emphasized that although Hatfield and Milgrom and Kojima et al. (2014) provide useful toolkits for proving theoretical properties of DA-based mechanisms, developing a concrete mechanism that works for new types of constraints remains challenging; we need to appropriately design a choice function of schools so that their frameworks are applicable while the required design goals can be achieved. Also, the stability concept used in them is defined through choice functions. A choice function of a school takes a set of contracts, each of which is a student-school pair, as an argument, and decides which contracts the school should accept. If a student prefers a certain school/contract but it is not chosen by the school's choice function, her claim to assign her to the school is not considered legitimate. In our model, the choice function of a school takes into account distributional constraints in addition to its preference ranking. As a result, it is not obvious for a student why her contract is not considered legitimate, in particular, which constraint prevents her from being assigned to the school. To complement this, we define our stability requirements in a more detailed, explicit way. To be more precise, we classify the conditions where a student's claim is considered legitimate, i.e., either she has a justified envy towards another student, or she claims an empty seat of a school. We show that our stability concepts are actually equivalent to the stability concept provided by Hatfield and Milgrom, which we call Hatfield-Milgrom (HM)-stability. Thus, our stability requirements provide an alternative interpretation of HM-stability.

2. Fleiner (2003) obtains some of the results including the existence of a stable allocation in a framework that is more general than the model in the work of Hatfield and Milgrom (2005). However, he does not show results regarding incentives, which are important for our purposes. 
Alternatively, Goto, Kojima, Kurata, Tamura, and Yokoo (2016) present a general framework for handling more unrestricted class of constraints (beyond $\mathrm{M}^{\mathrm{h}}$-concavity) and a class of strategy-proof mechanism that is different from the generalized DA mechanism. Our DA-OT is not an instance of their mechanism; it is an instance of the generalized DA mechanism. Kamada and Kojima (2014) also provide a general framework for handling hierarchical regional maximum quotas. Furthermore, Yokoi (2014) proposes yet another general framework, in which each school has a matroidal choice function. Currently, we are not sure whether we can utilize these two frameworks in our setting, since their models look significantly different from ours.

\section{Model for Controlled School Choice Program}

We first show a basic model that is a straightforward extension of the one presented by Ehlers et al. (2014). We then consider a slightly modified model for our mechanisms.

\subsection{Basic Model}

A market is a tuple $\left(S, C, T, \tau, X, \succ_{S}, \succ_{C}, q_{C}, p_{C, T}\right)$, where each component is defined as follows:

- finite number of students $S=\left\{s_{1}, s_{2}, \ldots, s_{n}\right\}$,

- finite number of schools $C=\left\{c_{1}, c_{2}, \ldots, c_{m}\right\}$,

- type space $T=\left\{t_{1}, t_{2}, \ldots, t_{k}\right\}$,

- type function $\tau: S \rightarrow 2^{T}$, where $\tau(s)$ is the set of types to which student $s$ belongs,

- set of contracts $X=S \times C$, where $(s, c) \in X$ means student $s$ is matched to school $c$,

- students' preference profile $\succ_{S}=\left(\succ_{s_{1}}, \ldots, \succ_{s_{n}}\right)$, where $\succ_{s}$ is the strict preference relation of student $s \in S$ over contracts related to $s$, i.e., $(s, c) \succ_{s}\left(s, c^{\prime}\right)$ means that student $s$ strictly prefers school $c$ over school $c^{\prime}$,

- schools' priority profile $\succ_{C}=\left(\succ_{c_{1}}, \ldots, \succ_{c_{m}}\right)$, where each $\succ_{c}$ is the strict priority ranking of school $c \in C$ over contracts related to $c$, i.e., $(s, c) \succ_{c}\left(s^{\prime}, c\right)$ means that student $s$ has a higher priority ranking than student $s^{\prime}$ to be enrolled at school $c$,

- vector of maximum quotas $q_{C}=\left(q_{c}\right)_{c \in C}$, where $q_{c}$ is the maximum quota (capacity) of school $c \in C$,

- vector of soft-bounds $p_{C, T}=\left(p_{c_{1}, T}, \ldots, p_{c_{m}, T}\right)$, where each $p_{c, T}=\left(p_{c, t_{1}}, \ldots, p_{c, t_{k}}\right)$ represents type-specific soft-bounds, i.e., $p_{c, t}$ is a (non-binding) target quota of students with type $t$ whom school $c$ is supposed to accept.

We assume $|\tau(s)| \geq 1$, i.e., a student can belong to multiple types. This is the only essential difference between our model and the one by Ehlers et al. (2014), which assumes $|\tau(s)|=1$.

To be more precise, the model by Ehlers et al. applies two parameters, i.e., a floor and a ceiling, that specify a non-binding target range. In this subsection, we use a simplified model with one parameter for each school and each type, since there is a chance that no stable matching exists even in this simplified model. In Section 5, we introduce an extended model that applies two parameters as Ehlers et al. did. 
For notational simplicity, we assume each contract $(s, c) \in X$ is acceptable for both $s$ and $c$. This assumption is not crucial, and the results obtained in this paper still hold when this assumption is relaxed, i.e., when a student (or a school) considers some schools (or students) unacceptable or a mechanism allows a student (or a school) being unmatched. We also assume $\sum_{c \in C} q_{c} \geq n$ holds, i.e., the total capacity of the schools is large enough to accept all the students. We assume $\sum_{t \in T} p_{c, t} \leq q_{c}$ holds for all $c \in C$, i.e., target quotas can be satisfied without violating the maximum quota. For $X^{\prime} \subseteq X$, let $X_{s}^{\prime}$ denote $\left\{(s, c) \in X^{\prime} \mid c \in C\right\}$, and let $X_{c}^{\prime}$ denote $\left\{(s, c) \in X^{\prime} \mid s \in S\right\}$. Also, let $X_{c, t}^{\prime}$ denote $\left\{(s, c) \in X^{\prime} \mid s \in S, t \in \tau(s)\right\}$.

We say $X^{\prime}$ is feasible if $\left|X_{s}^{\prime}\right|=1$ and $\left|X_{c}^{\prime}\right| \leq q_{c}$ hold for all $s \in S$ and $c \in C$. We call a feasible set of contracts a matching. Let $\mathcal{X}$ denote a set of matchings. $X^{\prime} \in \mathcal{X}$ is student-optimal within $\mathcal{X}$ if $X_{s}^{\prime} \succ_{s} X_{s}^{\prime \prime}$ or $X_{s}^{\prime}=X_{s}^{\prime \prime}$ holds for all $X^{\prime \prime} \in \mathcal{X}$ and $s \in S$. A mechanism is a function that takes a profile of students' preferences as input and returns matching $X^{\prime}$. We say a mechanism is strategy-proof if no student ever has any incentive to misreport her preference, regardless what the other students report.

Let us introduce two conditions that compose stability.

Definition 1 (justified envy for the basic model). We say student $s$ has justified envy toward $s^{\prime} \neq s$ in matching $X^{\prime}$, where $(s, c),\left(s^{\prime}, c^{\prime}\right) \in X^{\prime}$, if the following conditions hold: $\left(s, c^{\prime}\right) \succ_{s}(s, c),\left(s, c^{\prime}\right) \succ_{c^{\prime}}\left(s^{\prime}, c^{\prime}\right)$, and $\forall t \in \tau\left(s^{\prime}\right) \backslash \tau(s),\left|X_{c^{\prime}, t}^{\prime}\right|>p_{c^{\prime}, t}\left(\right.$ or $\left.\tau\left(s^{\prime}\right) \backslash \tau(s)=\emptyset\right)$.

Basically, student $s$ can have justified envy toward another student $s^{\prime}$, when $s$ would rather be matched to school $c^{\prime}$ than her current school $c$, and she has a higher priority ranking at $c^{\prime}$ than student $s^{\prime}$. However, if $s^{\prime}$ belongs to type $t$ (and $s$ does not), and the number of type $t$ students accepted to $c^{\prime}$ is less than or equal to $p_{c^{\prime}, t}$, the envy of $s$ toward $s^{\prime}$ cannot be justified. We say that matching $X^{\prime}$ is fair if no student has justified envy for the basic model. We say that a mechanism is fair if it always gives a fair matching.

Definition 2 (claiming an empty seat for the basic model). We say student s claims an empty seat of $c^{\prime}$ in matching $X^{\prime}$, where $(s, c) \in X^{\prime}$, if the following conditions hold: $\left(s, c^{\prime}\right) \succ_{s}(s, c)$ and $\left|X_{c^{\prime}}^{\prime}\right|<q_{c^{\prime}}$. Also, we say student s claims an empty seat of $c^{\prime}$ "by type" in matching $X^{\prime}$, where $(s, c) \in X^{\prime}$, if the following conditions hold: $\left(s, c^{\prime}\right) \succ_{s}(s, c)$ and $\exists t \in \tau(s),\left|X_{c^{\prime}, t}^{\prime}\right|<p_{c^{\prime}, t}$.

We say that matching $X^{\prime}$ is nonwasteful if no student claims an empty seat or claims an empty seat by type for the basic model. We say that a mechanism is nonwasteful if it always gives a nonwasteful matching. Also, we say that a matching is stable if it is fair and nonwasteful for the basic model. When $|\tau(s)|=1$ for all $s \in S$, our definition of stability becomes equivalent to fairness under the soft-bounds and nonwastefulness used by Ehlers et al. (2014).

Next, let us show a case where no stable matching exists.

Example 1. Assume $S=\left\{s_{1}, s_{2}, s_{3}, s_{4}\right\}, C=\left\{c_{1}, c_{2}, c_{3}\right\}, T=\left\{t_{1}, t_{2}, t_{3}\right\}, \tau\left(s_{1}\right)=\left\{t_{3}\right\}$, $\tau\left(s_{2}\right)=\left\{t_{1}, t_{2}\right\}, \tau\left(s_{3}\right)=\left\{t_{1}\right\}$, and $\tau\left(s_{4}\right)=\left\{t_{2}\right\}$. Also assume $q_{c_{1}}=2, p_{c_{1}, t_{1}}=1$, $p_{c_{1}, t_{2}}=1, p_{c_{1}, t_{3}}=0, q_{c_{2}}=1$, and $q_{c_{3}}=1$. We assume $p_{c_{2}, t}$ and $p_{c_{3}, t}$ are 0 for all t. The priorities of all schools are identical, i.e., $\succ_{c}:\left(s_{1}, c\right),\left(s_{2}, c\right),\left(s_{3}, c\right),\left(s_{4}, c\right)$. Also, $\succ_{s_{2}}:\left(s_{2}, c_{2}\right),\left(s_{2}, c_{1}\right),\left(s_{2}, c_{3}\right)$. The preferences of the other students are identical, i.e., $\succ_{s}$ : $\left(s, c_{1}\right),\left(s, c_{2}\right),\left(s, c_{3}\right)$. 
Let us show that there exists no stable matching in this situation. First, assume $s_{2}$ is assigned to $c_{1}$. If $s_{3}$ or $s_{4}$ is assigned to $c_{1}, s_{1}$ has justified envy. If no other student is assigned to $c_{1}, s_{1}$ claims an empty seat of $c_{1}$. $s_{1}$ together with $s_{2}$ must be assigned to $c_{1}$. Then, $s_{3}$ or $s_{4}$ must be assigned to $c_{2}$. However, since $s_{2}$ has justified envy toward the student assigned to $c_{2}, s_{2}$ cannot be assigned to $c_{1}$.

Next, assume $s_{2}$ is not assigned to $c_{1}$. Then both $s_{3}$ and $s_{4}$ are assigned to $c_{1}$. Otherwise, they can claim an empty seat by type. Assume $s_{2}$ is assigned to $c_{2} . s_{1}$ must be assigned to $c_{3}$. However, since $s_{1}$ has justified envy toward $s_{2}, s_{2}$ cannot be assigned to $c_{2}$. Assume $s_{2}$ is assigned to $c_{3}$. However, then $s_{2}$ has justified envy toward $s_{3}$ or $s_{4}$. If $s_{2}$ is not assigned to any school, there exists school $c$ whose maximum quota is not satisfied. $s_{2}$ claims an empty seat of $c$. Thus, there exists no matching that is fair and nonwasteful.

\subsection{New Basic Model}

The model is quite similar to the previous model. One major difference is as follows: in the basic model, soft bounds on multiple types can be met by the same student, while in the new model, a student can satisfy a soft bound on at most one type. To this end, school $c$ provides distinct reserved seats for each type, and each contract explicitly states that a student is assigned to a particular reserved seat of a school. As a result, to fulfill the quotas of multiple types, a school needs distinct students/contracts. The preferences/priorities of the students/schools, as well as the stability requirements, are defined based on these contracts.

To be more precise, we represent a market as a tuple $\left(S, C, T, \tau, X, \succ_{S}, \succ_{C}, q_{C}, p_{C, T}\right)$. The definitions of $S, C, T, \tau$, and $q_{C}$ are identical to the previous model. School $c$ provides distinct reserved seats for each type. Contract $x \in X$ is represented as $(s, c, t)$, which describes the fact that student $s$ is assigned to type $t$ seat of school $c$. Thus, $X$ is given as $\{(s, c, t) \mid s \in S, c \in C, t \in \tau(s)\}$.

Since we assume student $s$ has a strict preference over contracts related to her, she has a preference over seats of the same school. This assumption is natural if we assume a school provides a different program for different seats, e.g., a student who is assigned to a "financially-distressed" seat can obtain a scholarship, and a student who is assigned to an "English as a second language" seat can attend an English language class. Also, this definition allows a case such that $(s, c, t) \succ_{s}\left(s, c^{\prime}, t\right) \succ_{s}\left(s, c, t^{\prime}\right) \succ_{s}\left(s, c^{\prime}, t^{\prime}\right)$, i.e., student $s$ prefers type $t$ seat over type $t^{\prime}$ seat, and if the types of seats are the same, she prefers school $c$ over school $c^{\prime} .^{3}$

We also assume school $c$ has a strict priority ranking over contracts related to it. When both $s$ and $s^{\prime}$ have types $t$ and $t^{\prime}$, there is a chance that $(s, c, t) \succ_{c}\left(s^{\prime}, c, t\right)$ and $\left(s^{\prime}, c, t^{\prime}\right) \succ_{c}$ $\left(s, c, t^{\prime}\right)$ hold, i.e., the relative ordering of $s$ and $s^{\prime}$ can be different for different seats. This assumption is also natural, e.g., for "financially-distressed" seats, $s$ has a higher priority ranking than $s^{\prime}$ since $s$ is more financially distressed, while for "standard" seats, $s^{\prime}$ has a higher priority ranking than $s$ since $s^{\prime}$ has a better SAT score.

3. If a student is indifferent between different seats of the same school, we can break the ties arbitrarily to form a strict preference and apply our mechanism, which is still strategy-proof and obtains a stable matching. However, we can no longer guarantee student-optimality. See the work by Abdulkadiroğlu, Pathak, and Roth (2009) for discussion on handling indifference in students' preferences. 
For any subset of contracts $X^{\prime} \subseteq X$, let $X_{s}^{\prime}$ denote $\left\{(s, c, t) \in X^{\prime} \mid c \in C, t \in T\right\}$, and let $X_{c}^{\prime}$ denote $\left\{(s, c, t) \in X^{\prime} \mid s \in S, t \in T\right\}$. Also, let $X_{c, t}^{\prime}$ denote $\left\{(s, c, t) \in X^{\prime} \mid s \in S\right\}$. Note that $\left|X_{c, t}^{\prime}\right|$ means the number of type $t$ students accepted to $c$ in the previous model. Here $\left|X_{c, t}^{\prime}\right|$ means the number of students accepted to type $t$ seats of school $c$. The actual number of type $t$ students accepted to $c$ can exceed $\left|X_{c, t}^{\prime}\right|$, since a student who has another type (as well as $t$ ) might be assigned to a different seat of school $c$.

The definition of feasibility in this model is identical to the existing model. We modify the definitions of justified envy and claiming an empty seat as follows.

Definition 3 (justified envy for the new basic model). We say student $s$ has justified envy toward $s^{\prime} \neq s$ in matching $X^{\prime}$, where $(s, c, t),\left(s^{\prime}, c^{\prime}, t^{\prime}\right) \in X^{\prime}$ and $\left(s, c^{\prime}, t^{\prime \prime}\right) \in X \backslash X^{\prime}$, if the following conditions hold: $\left(s, c^{\prime}, t^{\prime \prime}\right) \succ_{s}(s, c, t),\left(s, c^{\prime}, t^{\prime \prime}\right) \succ_{c^{\prime}}\left(s^{\prime}, c^{\prime}, t^{\prime}\right)$, and either (fr-i) $t^{\prime}=t^{\prime \prime}$ or $(\boldsymbol{f} r-i \boldsymbol{i})\left|X_{c^{\prime}, t^{\prime}}^{\prime}\right|>p_{c^{\prime}, t^{\prime}}$.

As in the existing definition, basically, student $s$ can have justified envy toward another student $s^{\prime}$, when $s$ prefers $\left(s, c^{\prime}, t^{\prime \prime}\right)$ over her current contract, and $\left(s, c^{\prime}, t^{\prime \prime}\right)$ has a higher priority ranking at $c^{\prime}$ than $\left(s^{\prime}, c^{\prime}, t^{\prime}\right)$. However, if $t^{\prime} \neq t^{\prime \prime}$ and the number of type $t^{\prime}$ contracts accepted to $c^{\prime}$ is less than or equal to $p_{c^{\prime}, t^{\prime}}$, this envy cannot be justified.

Definition 4 (claiming an empty seat for the new basic model). We say student s claims an empty seat of $c^{\prime}$ in matching $X^{\prime}$, where $(s, c, t) \in X^{\prime}$ and $\left(s, c^{\prime}, t^{\prime}\right) \in X \backslash X^{\prime}$, if $\left(s, c^{\prime}, t^{\prime}\right) \succ_{s}$ $(s, c, t)$, and one of the following conditions holds:

$\left(\right.$ nw-i) $\left|X_{c^{\prime}}^{\prime}\right|<q_{c^{\prime}}$, or

(nw-ii) $c=c^{\prime},\left(s, c, t^{\prime}\right) \succ_{c}(s, c, t)$, and $\left|X_{c, t}^{\prime}\right|>p_{c, t}$.

We say student $s$ claims an empty seat of $c^{\prime}$ by type in matching $X^{\prime}$, where $(s, c, t) \in X^{\prime}$ and $\left(s, c^{\prime}, t^{\prime}\right) \in X \backslash X^{\prime}$, if $\left(s, c^{\prime}, t^{\prime}\right) \succ_{s}(s, c, t)$ and the following condition holds:

(nw-iii) $\left|X_{c^{\prime}, t^{\prime}}^{\prime}\right|<p_{c^{\prime}, t^{\prime}}$.

Conditions (nw-i) and (nw-iii) are basically identical to the original definition. Condition (nw-ii) can be considered as $s$ having justified envy toward her own contract $(s, c, t)$, which is accepted while she prefers $\left(s, c, t^{\prime}\right)$ and it has a higher priority ranking at $c$ than $(s, c, t)$.

One main reason that, for the basic model, no stable (fair and nonwasteful) matching exists in the previous example is that $s_{1}$ and $s_{2}$ are complementary for $c_{1}$, i.e., if $c_{1}$ accepts $s_{2}$, it also needs to accept $s_{1}$, while if $c_{1}$ does not accept $s_{2}$, it cannot accept $s_{1} \cdot{ }^{4}$ By introducing type-specific contracts, such a complementary relation disappears. Accepting contract $\left(s_{2}, c_{1}, t_{1}\right)$ (or $\left.\left(s_{2}, c_{1}, t_{2}\right)\right)$ does not give any advantage for $\left(s_{1}, c_{1}, t_{3}\right)$ to be accepted. As shown in the next section, for the new basic model, a stable (i.e., fair and nonwasteful) matching always exists.

4. Note that the substitutability is not a necessary condition for the existence of a stable matching. There exist models with complementarities where the existence of a stable matching is guaranteed (see, for example, Hatfield \& Kojima, 2008). 


\section{Deferred Acceptance Mechanism for Overlapping Types}

In this section we propose a strategy-proof mechanism called Deferred Acceptance mechanism for Overlapping Types (DA-OT).

This mechanism is built upon choice functions $C h_{S}: 2^{X} \rightarrow 2^{X}$ and $C h_{C}: 2^{X} \rightarrow 2^{X}$. $C h_{S}$ is a choice function of students $S$, which is a union of individual choice functions, i.e., $C h_{S}\left(X^{\prime}\right):=\bigcup_{s \in S} C h_{s}\left(X^{\prime}\right)$. In the same way, $C h_{C}$ is a choice function of schools $C$, which is a union of individual choice functions, i.e., $C h_{C}\left(X^{\prime}\right):=\bigcup_{c \in C} C h_{c}\left(X^{\prime}\right)$. The choice function of student $s$ is simple. $C h_{s}\left(X^{\prime}\right)$ returns $(s, c, t)$, which is $s$ 's most preferred contract in $X_{s}^{\prime}$ (or $\emptyset$ if $X_{s}^{\prime}$ is $\emptyset$ ). The choice function of school $c$ for DA-OT is more complicated and is defined as follows. Here, $Y_{c, t}:=\{(s, c, t) \in Y \mid s \in S\}$ and $Z_{c, t}:=\{(s, c, t) \in Z \mid s \in S\}$.

Definition 5 (Choice function of school $c$ for DA-OT). $C h_{c}\left(X^{\prime}\right)$ is defined as follows:

Step $1 Y \leftarrow \emptyset, Z \leftarrow X_{c}^{\prime}$.

Step 2 For each $t \in T$, repeat the following procedure: If $\left|Y_{c, t}\right|=p_{c, t}$ or $\left|Z_{c, t}\right|=0$ then skip to the iteration for the next $t$. Otherwise, choose $(s, c, t) \in Z$ with the highest priority ranking in $Z$ based on $\succ_{c}$. Move $(s, c, t)$ from $Z$ to $Y$.

Step 3 Repeat the following procedure: If $|Y|=q_{c}$ or $|Z|=0$, return $Y$. Otherwise, choose $(s, c, t) \in Z$ with the highest priority ranking in $Z$ based on $\succ_{c}$. Move $(s, c, t)$ from $Z$ to $Y$.

Note that $Y_{c, t}$ in Step 2 corresponds to the set of reserved seats of type $t$ and school c. DA-OT is one instance of the student-proposing generalized DA mechanism presented by Hatfield and Milgrom (2005). The students and schools declare their preferences and priorities before the mechanism begins. Let us define DA-OT as follows.

Definition 6 (Deferred Acceptance mechanism for Overlapping Types (DA-OT)).

Step $1 R e \leftarrow \emptyset$.

Step $2 X^{\prime} \leftarrow C h_{S}(X \backslash R e), X^{\prime \prime} \leftarrow C h_{C}\left(X^{\prime}\right)$

Step 3 If $X^{\prime}=X^{\prime \prime}$, then return $X^{\prime}$, otherwise, $R e \leftarrow R e \cup\left(X^{\prime} \backslash X^{\prime \prime}\right)$, go to Step 2.

Here Re represents the rejected contracts. A student cannot choose a contract in Re. First, students propose a set of contracts that are most preferred and not rejected so far, namely $X^{\prime}$. Then schools choose $X^{\prime \prime}$, which is a subset of $X^{\prime}$. If no contract is rejected, the mechanism terminates. Otherwise, the rejected contracts are added to $R e$, and the mechanism repeats the same procedure.

We show an example to illustrate the execution of DA-OT.

Example 2. We consider a situation that is basically identical to Example 1. We assume the preference of $s_{2}$ is given as:

$$
\begin{array}{rlll}
\succ_{s_{2}}: & \left(s_{2}, c_{2}, t_{1}\right), & \left(s_{2}, c_{2}, t_{2}\right), & \left(s_{2}, c_{1}, t_{1}\right), \\
& \left(s_{2}, c_{1}, t_{2}\right), & \left(s_{2}, c_{3}, t_{1}\right), & \left(s_{2}, c_{3}, t_{2}\right) .
\end{array}
$$


The preferences of the other students are basically identical, i.e.,

$$
\begin{array}{llll}
\succ_{s_{1}}: & \left(s_{1}, c_{1}, t_{3}\right), & \left(s_{1}, c_{2}, t_{3}\right), & \left(s_{1}, c_{3}, t_{3}\right), \\
\succ_{s_{3}}: & \left(s_{3}, c_{1}, t_{1}\right), & \left(s_{3}, c_{2}, t_{1}\right), & \left(s_{3}, c_{3}, t_{1}\right), \\
\succ_{s_{4}}: & \left(s_{4}, c_{1}, t_{2}\right), & \left(s_{4}, c_{2}, t_{2}\right), & \left(s_{4}, c_{3}, t_{2}\right) .
\end{array}
$$

Also, the preferences of all schools are identical and are defined as

$$
\begin{array}{rll}
\succ_{c}: & \left(s_{1}, c, t_{3}\right), & \left(s_{2}, c, t_{1}\right), \quad\left(s_{2}, c, t_{2}\right), \\
& \left(s_{3}, c, t_{1}\right), \quad\left(s_{4}, c, t_{2}\right) .
\end{array}
$$

First, each student chooses her most preferred contract;

$$
X^{\prime}=\left\{\left(s_{1}, c_{1}, t_{3}\right),\left(s_{2}, c_{2}, t_{1}\right),\left(s_{3}, c_{1}, t_{1}\right),\left(s_{4}, c_{1}, t_{2}\right)\right\} .
$$

$C h_{c_{1}}\left(X^{\prime}\right)$ is $\left\{\left(s_{3}, c_{1}, t_{1}\right),\left(s_{4}, c_{1}, t_{2}\right)\right\}$. $\left(s_{1}, c_{1}, t_{3}\right)$, which has the highest priority ranking, is rejected since $\left(s_{3}, c_{1}, t_{1}\right)$ and $\left(s_{4}, c_{1}, t_{2}\right)$ are selected at Step 2 in Definition 5.

Then $s_{1}$ chooses her second preferred contract;

$$
X^{\prime}=\left\{\left(s_{1}, c_{2}, t_{3}\right),\left(s_{2}, c_{2}, t_{1}\right),\left(s_{3}, c_{1}, t_{1}\right),\left(s_{4}, c_{1}, t_{2}\right)\right\} .
$$

$C h_{c_{2}}\left(X^{\prime}\right)$ is $\left\{\left(s_{1}, c_{2}, t_{3}\right)\right\}$, since $\left(s_{1}, c_{2}, t_{3}\right)$ has a higher priority ranking than $\left(s_{2}, c_{2}, t_{1}\right)$.

Next $s_{2}$ chooses her second preferred contract, i.e., $\left(s_{2}, c_{2}, t_{2}\right)$, but this contract is also rejected. Thus, $s_{2}$ chooses her third preferred contract, i.e., $\left(s_{2}, c_{1}, t_{1}\right)$;

$$
X^{\prime}=\left\{\left(s_{1}, c_{2}, t_{3}\right),\left(s_{2}, c_{1}, t_{1}\right),\left(s_{3}, c_{1}, t_{1}\right),\left(s_{4}, c_{1}, t_{2}\right)\right\} .
$$

$C h_{c_{1}}\left(X^{\prime}\right)$ is $\left\{\left(s_{2}, c_{1}, t_{1}\right),\left(s_{4}, c_{1}, t_{2}\right)\right\}$, since $\left(s_{2}, c_{2}, t_{1}\right)$ has a higher priority ranking than $\left(s_{3}, c_{1}, t_{1}\right)$.

Then $s_{3}$ chooses her second preferred contract;

$$
X^{\prime}=\left\{\left(s_{1}, c_{2}, t_{3}\right),\left(s_{2}, c_{1}, t_{1}\right),\left(s_{3}, c_{2}, t_{1}\right),\left(s_{4}, c_{1}, t_{2}\right)\right\} \text {. }
$$

$C h_{c_{2}}\left(X^{\prime}\right)$ is $\left\{\left(s_{1}, c_{2}, t_{3}\right)\right\}$, since $\left(s_{1}, c_{2}, t_{3}\right)$ has a higher priority ranking than $\left(s_{3}, c_{2}, t_{1}\right)$.

Finally, $s_{3}$ chooses her third preferred contract;

$$
X^{\prime}=\left\{\left(s_{1}, c_{2}, t_{3}\right),\left(s_{2}, c_{1}, t_{1}\right),\left(s_{3}, c_{3}, t_{1}\right),\left(s_{4}, c_{1}, t_{2}\right)\right\} .
$$

No contract is rejected and the mechanism terminates.

Note that in the original basic model, $s_{1}$ has justified envy toward $s_{4}$ because $c_{1}$ 's target quotas are satisfied by accepting $s_{2}$. This is not the case in our new model, since $s_{2}$ is accepted for a type $t_{1}$ seat and $s_{4}$ is accepted for a type $t_{2}$ seat.

\section{Properties of DA-OT}

In this section, we first introduce another stability condition called Hatfield-Milgrom (HM)stability (Hatfield \& Milgrom, 2005). 
Definition 7 (HM-stability). We say matching $X^{\prime}$ is HM-stable if $X^{\prime}=C h_{S}\left(X^{\prime}\right)=$ $C h_{C}\left(X^{\prime}\right)$ and there exists no $x \in X \backslash X^{\prime}$ such that $x \in C h_{S}\left(X^{\prime} \cup\{x\}\right)$ and $x \in C h_{C}\left(X^{\prime} \cup\{x\}\right)$.

Next, we show that HM-stability is equivalent to our stability (Lemma 1 in Appendix A.1). It is shown that if $C h_{C}$ satisfies three properties, i.e., the irrelevance of rejected contracts, the law of aggregate demand, and the substitutes condition, then a HM-stable matching always exists. Also, the generalized DA mechanism is strategy-proof and obtains the student-optimal matching among all the HM-stable matchings. We show that $C h_{C}$ satisfies these properties (Lemma 2 in Appendix A.1). From these lemmas, we obtain our main theorem.

Theorem 1. A stable matching always exists, and DA-OT is strategy-proof and obtains a stable matching, which is student-optimal among all the stable matchings.

Proof. Hatfield and Milgrom (2005) show that when $C h_{C}$ satisfies the three properties, a HM-stable matching always exists and the generalized DA mechanism is strategy-proof and obtains the student-optimal matching among all the HM-stable matchings. HM-stability is equivalent to our stability (Lemma 1). DA-OT is one instance of the generalized DA mechanism. Also, $C h_{C}$ satisfies these properties (Lemma 2).

Recall that Kojima (2012) shows in his model with two types (majority and minority), it is possible that setting a hard bound to majority students can hurt all minority students. Hafalir et al. (2013) show that with soft bounds, this will not happen, i.e., by increasing the soft bound of one particular school $c^{*}$ and one particular type $t^{*}$, at least one type $t^{*}$ student weakly prefers the outcome when the soft bound is increased. We can derive a similar result in our setting, i.e., the following theorem holds.

Theorem 2. Consider DA-OT with $p_{C, T}$ and $p_{C, T}^{\prime}$. Here, $p_{C, T}$ and $p_{C, T}^{\prime}$ are almost identical, i.e., $p_{c, t}=p_{c, t}^{\prime}$ holds except for one particular school $c^{*}$ and one particular type $t^{*}$. We assume $p_{c^{*}, t^{*}}<p_{c^{*}, t^{*}}^{\prime}$ holds. For a given preference profile, let $X^{\prime}$ and $Y^{\prime}$ be the set of contracts obtained with $p_{C, T}$ and $p_{C, T}^{\prime}$, respectively. Then, there exists at least one student $s$ where $t^{*} \in \tau(s)$ such that $Y_{s}^{\prime} \succeq_{s} X_{s}^{\prime}$ holds.

Note that $Y_{s}^{\prime} \succeq_{s} X_{s}^{\prime}$ indicates $Y_{s}^{\prime} \succ_{s} X_{s}^{\prime}$ or $Y_{s}^{\prime}=X_{s}^{\prime}$. The proof is placed in Appendix A.2.

Theorem 3. The time complexity of DA-OT is $O\left(|X|^{3}\right)$, where $X$ is the set of all contracts.

Proof. Since Re in Definition 6 expands monotonically, Step 2 is repeated at most $|X|$ times. The time required to calculate $C h_{S}\left(X^{\prime}\right)$ or $C h_{C}\left(X^{\prime}\right)$ is at most $O\left(\left|X^{\prime}\right|^{2}\right)$. Overall, the time complexity of DA-OT is $O\left(|X|^{3}\right)$.

About the influence of $|T|$ (the size of the type space) toward the computation time, we can assume $|X|$ is (at most) linear in $|T|$. Thus, the time complexity is polynomial in $|T|$.

\section{Handling Type-Specific Floor/Ceiling}

In this section, we introduce an extended model that applies two parameters, i.e., a floor and a ceiling, that specify a non-binding target range. A similar model is used by Ehlers et al. (2014). 


\subsection{Extended Model}

We represent a market as a tuple $\left(S, C, T, \tau, X, \succ_{S}, \succ_{C}, q_{C}, p_{C, T}, q_{C, T}\right)$. Here, the only additional element from the new basic model is the vector of soft-bounds $q_{C, T}=\left(q_{c_{1}, T}, \ldots, q_{c_{m}, T}\right)$, where each $q_{c, T}=\left(q_{c, t_{1}}, \ldots, q_{c, t_{k}}\right)$ represents type-specific ceilings, i.e., $q_{c, t}$ is a (non-binding) target quota, such that school $c$ should limit the number of students with type $t$. We assume for all $c \in C, t \in T, p_{c, t} \leq q_{c, t}$ holds.

As in the basic model, these soft-bounds do not affect feasibility. We modify Definitions 3 and 4 as follows.

Definition 8 (justified envy for the extended model). We say student s has justified envy toward $s^{\prime} \neq s$ in matching $X^{\prime}$, where $(s, c, t),\left(s^{\prime}, c^{\prime}, t^{\prime}\right) \in X^{\prime}$ and $\left(s, c^{\prime}, t^{\prime \prime}\right) \in X \backslash X^{\prime}$, if the following conditions hold: $\left(s, c^{\prime}, t^{\prime \prime}\right) \succ_{s}(s, c, t),\left(s, c^{\prime}, t^{\prime \prime}\right) \succ_{c^{\prime}}\left(s^{\prime}, c^{\prime}, t^{\prime}\right)$, and one of the following conditions holds:

(fr-i) $t^{\prime}=t^{\prime \prime}$,

(fr-ii) $\left|X_{c^{\prime}, t^{\prime}}^{\prime}\right|>p_{c^{\prime}, t^{\prime}}$ and $\left|X_{c^{\prime}, t^{\prime \prime}}^{\prime}\right|<q_{c^{\prime}, t^{\prime \prime}}$, or

(fr-iii) $\left|X_{c^{\prime}, t^{\prime}}^{\prime}\right|>q_{c^{\prime}, t^{\prime}}$ and $\left|X_{c^{\prime}, t^{\prime \prime}}^{\prime}\right| \geq q_{c^{\prime}, t^{\prime \prime}}$

Basically, student $s$ can have justified envy toward another student $s^{\prime}$, when $s$ prefers $\left(s, c^{\prime}, t^{\prime \prime}\right)$ over her current contract, and $\left(s, c^{\prime}, t^{\prime \prime}\right)$ has a higher priority ranking at $c^{\prime}$ than $\left(s^{\prime}, c^{\prime}, t^{\prime}\right)$. However, if $t^{\prime} \neq t^{\prime \prime}$ and the number of type $t^{\prime}$ contracts accepted by $c^{\prime}$ is less than or equal to $p_{c^{\prime}, t^{\prime}}$, or the number of type $t^{\prime \prime}$ contracts accepted by $c^{\prime}$ is more than or equal to $q_{c^{\prime}, t^{\prime \prime}}$, while the number of type $t^{\prime}$ contracts accepted by $c^{\prime}$ is less than or equal to $q_{c^{\prime}, t^{\prime}}$, this envy cannot be justified. We say that matching $X^{\prime}$ is fair if no student has justified envy for the extended model. We say a mechanism is fair if it always gives a fair matching.

Definition 9 (claiming an empty seat for the extended model). We say student $s$ claims an empty seat of $c^{\prime}$ in matching $X^{\prime}$, where $(s, c, t) \in X^{\prime}$ and $\left(s, c^{\prime}, t^{\prime}\right) \in X \backslash X^{\prime}$, if $\left(s, c^{\prime}, t^{\prime}\right) \succ_{s}$ $(s, c, t)$, and one of the following conditions holds:

(nw-i) $\left|X_{c^{\prime}}^{\prime}\right|<q_{c^{\prime}}$,

(nw-ii) $c=c^{\prime},\left(s, c, t^{\prime}\right) \succ_{c}(s, c, t)$, and $\left|X_{c, t}^{\prime}\right|>p_{c, t}$, and $\left|X_{c, t^{\prime}}^{\prime}\right|<q_{c, t^{\prime}}$, or

(nw-iii) $c=c^{\prime},\left(s, c, t^{\prime}\right) \succ_{c}(s, c, t)$, and $\left|X^{\prime}{ }_{c, t}\right|>q_{c, t}$, and $\left|X_{c, t^{\prime}}^{\prime}\right| \geq q_{c, t^{\prime}}$.

We say student $s$ claims an empty seat of $c^{\prime}$ by type in matching $X^{\prime}$, where $(s, c, t) \in X^{\prime}$ and $\left(s, c^{\prime}, t^{\prime}\right) \in X \backslash X^{\prime}$, if $\left(s, c^{\prime}, t^{\prime}\right) \succ_{s}(s, c, t)$ and one of the following conditions holds:

(nw-iv) $\left|X_{c^{\prime}, t^{\prime}}^{\prime}\right|<p_{c^{\prime}, t^{\prime}}$.

(nw-v) $p_{c^{\prime}, t^{\prime}} \leq\left|X_{c^{\prime}, t^{\prime}}^{\prime}\right|<q_{c^{\prime}, t^{\prime}}$ and there exists $t^{\prime \prime}$ such that $\left|X_{c^{\prime}, t^{\prime \prime}}^{\prime}\right|>q_{c^{\prime}, t^{\prime \prime}}$.

Conditions (nw-i) and (nw-iv) are identical to the previous definition. Conditions (nwii) and (nw-iii) can be considered as $s$ having justified envy toward her own contract $(s, c, t)$, which is accepted while she prefers $\left(s, c, t^{\prime}\right)$ and it has a higher priority ranking at $c$ than $(s, c, t)$. Condition (nw-v) means that school $c^{\prime}$ should respect the type-specific ceiling of 
type $t^{\prime \prime}$ since there exists another contract $\left(s, c^{\prime}, t^{\prime}\right)$, where type $t^{\prime}$ does not reach its ceiling yet. We say that matching $X^{\prime}$ is nonwasteful if no student claims an empty seat or claims an empty seat by type for the extended model and that a mechanism is nonwasteful if it always gives a nonwasteful matching. We say a matching is stable if it is fair and nonwasteful.

Note that each $q_{c, t}$ is just a soft ceiling and can be violated as long as the stability conditions are satisfied and the hard maximum quota for the school is satisfied. For Condition (fr-iii), the soft ceiling of school $c^{\prime}$ is already violated at type $t^{\prime}$. Then, the student $s$ can demand that the violation should happen at type $t^{\prime \prime}$, i.e., instead of accepting $\left(s^{\prime}, c^{\prime}, t^{\prime}\right)$, school $c^{\prime}$ should accept $\left(s, c^{\prime}, t^{\prime \prime}\right)$. For Condition (nw-iii), the soft ceiling of school $c=c^{\prime}$ is already violated at type $t$. Then, the student $s$ can demand that the violation should happen at type $t^{\prime}$, student, i.e., instead of accepting $(s, c, t)$, school $c$ should accept $\left(s, c, t^{\prime}\right)$. For Condition (nw-v), the spirit of type-specific ceiling $q_{c^{\prime}, t^{\prime \prime}}$ is that, school $c^{\prime}$ can violate $q_{c^{\prime}, t^{\prime \prime}}$ only if no student is rejected for another type $t^{\prime}$ while $q_{c^{\prime}, t^{\prime}}$ is not reached yet. Here, student $s$ wants to be accepted as type $t^{\prime}$ student, while $q_{c^{\prime}, t^{\prime}}$ is not reached yet. Thus, to respect the spirit of type-specific ceiling, school $c^{\prime}$ should reject some type $t^{\prime \prime}$ contract and accept $\left(s, c^{\prime}, t^{\prime}\right)$.

\subsection{Fine-Grained Model}

To simplify the description of our mechanism, we introduce a fine-grained model that utilizes contract types. More specifically, we represent a market as $\left(S, C, T, \tau, \tilde{X}, \tilde{\succ}_{S}, \tilde{\succ}_{C}, q_{C}, p_{C, T}\right.$, $\left.q_{C, T}\right)$. We assume there exist three different classes of seats for each type. There are $p_{c, t}$ first-class seats (where $k=1$ ), $q_{c, t}-p_{c, t}$ second-class seats (where $k=2$ ), and $q_{c}-q_{c, t}$ thirdclass seats (where $k=3$ ). Here, $\tilde{X}, \tilde{\succ}_{S}$, and $\tilde{\succ}_{C}$ are modified from the original extended model. Contract $x \in \tilde{X}$ is represented as $(s, c, t, k)$, which describes the fact that student

$s$ is assigned to type $t$ 's $k$-th class seat of school $c$, where $k \in\{1,2,3\} . \tilde{\succ}_{s}$ is obtained from $\succ_{s}$ such that $(s, c, t, k) \tilde{\succ}_{s}\left(s, c^{\prime}, t^{\prime}, k\right)$ holds iff $(s, c, t) \succ_{s}\left(s, c^{\prime}, t^{\prime}\right)$ holds, and for $k \neq k^{\prime}$, $(s, c, t, k) \tilde{\succ}_{s}\left(s, c, t, k^{\prime}\right)$ holds iff $k<k^{\prime}$ holds. In words, $\tilde{\succ}_{s}$ preserves the ordering in $\succ_{s}$. Furthermore, student $s$ prefers a first-class seat over a second-class seat, and a second-class seat over a third-class seat for the contracts of the same school and the same type. $\tilde{\succ}_{c}$ is obtained from $\succ_{c}$ such that $(s, c, t, k) \tilde{\succ}_{c}\left(s^{\prime}, c, t^{\prime}, k\right)$ holds iff $(s, c, t) \succ_{c}\left(s^{\prime}, c, t^{\prime}\right)$ holds, and for $k \neq k^{\prime},(s, c, t, k) \tilde{\succ}_{c}\left(s^{\prime}, c, t^{\prime}, k^{\prime}\right)$ holds iff $k<k^{\prime}$ holds. In words, $\tilde{\succ}_{c}$ preserves the ordering in $\succ_{c}$. Furthermore, school $c$ prefers the contracts of its first-class seats over that of its second-class seats, and the contracts of its second-class seats over that of its third-class seats. For $\tilde{X}^{\prime}$, we define $\tilde{X}_{c}^{\prime}$ and $\tilde{X}_{c, t}^{\prime}$ in a similar way to $X^{\prime}$. Furthermore, let $\tilde{X}_{c, t, k}^{\prime}$ denote $\left\{(s, c, t, k) \mid(s, c, t, k) \in \tilde{X}^{\prime}, s \in S\right\}$.

We define a mapping between $X^{\prime}$ in the original extended model and $\tilde{X}^{\prime}$ in the finegrained model as follows. The mapping from it to the original extended model is simple: for each $(s, c, t, k) \in \tilde{X}^{\prime}$, there exists corresponding contract $(s, c, t) \in X^{\prime}$. Let us define the mapping from the original extended model to the fine-grained model as follows. For each $(s, c, t) \in X^{\prime}$, if $(s, c, t)$ is within the top $p_{c, t}$ contracts in $X_{c, t}^{\prime}$ according to $\succ_{c}$, there exists a corresponding contract $(s, c, t, 1) \in \tilde{X}^{\prime}$. Otherwise, if $(s, c, t)$ is within the top $q_{c, t}$ contracts in $X_{c, t}^{\prime}$ according to $\succ_{c}$, there exists a corresponding contract $(s, c, t, 2) \in \tilde{X}^{\prime}$. Otherwise, there exists a corresponding contract $(s, c, t, 3) \in \tilde{X}^{\prime}$. 
We say matching $\tilde{X}^{\prime}$ in the fine-grained model is valid if there exists matching $X^{\prime}$ in the original extended model that is mapped to $\tilde{X}^{\prime}$. More specifically, if there exists a contract for the second-class seats for type $t$ of school $c,\left|\tilde{X}_{c, t, 1}^{\prime}\right|=p_{c, t}$ holds, i.e., there must exist exactly $p_{c, t}$ contracts for first-class seats. Also, if there exists a contract for the third-class seats for type $t$ of school $c,\left|\tilde{X}_{c, t, 1}^{\prime}\right|=p_{c, t}$ and $\left|\tilde{X}_{c, t, 2}^{\prime}\right|=q_{c, t}-p_{c, t}$ hold, i.e., there must exist exactly $p_{c, t}$ contracts for first-class seats and $q_{c, t}-p_{c, t}$ contracts for second-class seats.

\subsection{Mechanism}

In this subsection, we show how to modify DA-OT to handle type-specific ceilings in the finegrained model. We call the modified mechanism DA-OT*. DA-OT* is basically identical to DA-OT, but school's choice function $C h_{c}$ for DA-OT* is modified as follows. Here, for a set of contracts $Z_{c}^{k}$ (where $\left.k \in\{1,2,3\}\right), Z_{c, t}^{k}$ denotes $\left\{(s, c, t, k) \mid(s, c, t, k) \in Z^{k}, s \in S\right\}$.

Definition 10 (Choice function of school $c$ for DA-OT*). $C h_{c}\left(\tilde{X}^{\prime}\right)$ is defined as follows:

Step $1 Y \leftarrow \emptyset, Z_{c}^{1} \leftarrow\left\{(s, c, t, 1) \mid(s, c, t, 1) \in \tilde{X}^{\prime}, s \in S, t \in T\right\}, Z_{c}^{2} \leftarrow\{(s, c, t, 2) \mid$ $\left.(s, c, t, 2) \in \tilde{X}^{\prime}, s \in S, t \in T\right\}, Z_{c}^{3} \leftarrow\left\{(s, c, t, 3) \mid(s, c, t, 3) \in \tilde{X}^{\prime}, s \in S, t \in T\right\}$.

Step 2 For each $t \in T$, repeat the following procedure: If $\left|Y_{c, t, 1}\right|=p_{c, t}$ or $\left|Z_{c, t}^{1}\right|=0$ then skip to the iteration for the next $t$. Otherwise, choose $(s, c, t, 1) \in Z_{c}^{1}$ with the highest priority ranking in $Z_{c}^{1}$ based on $\tilde{\succ}_{c}$. Move $(s, c, t, 1)$ from $Z_{c}^{1}$ to $Y$.

Step 3 Repeat the following procedure: If $|Y|=q_{c}$ then return $Y$. If $\left|Z_{c}^{2}\right|=0$ then go to Step 4. Otherwise, choose $(s, c, t, 2) \in Z_{c}^{2}$ such that it has the highest priority ranking in $Z_{c}^{2}$ based on $\tilde{\succ}_{c}$ and it is not chosen yet in this step. If $\left|Y_{c, t, 2}\right| \neq q_{c, t}-p_{c, t}$ then move $(s, c, t, 2)$ from $Z_{c}^{2}$ to $Y$.

Step 4 Repeat the following procedure: If $|Y|=q_{c}$ or $\left|Z_{c}^{3}\right|=0$, return $Y$. Otherwise, choose $(s, c, t, 3) \in Z_{c}^{3}$ with the highest priority ranking in $Z_{c}^{3}$ based on $\tilde{\succ}_{c}$. Move $(s, c, t, 3)$ from $Z_{c}^{3}$ to $Y$.

\subsection{Properties}

This subsection shows that HM-stability of a matching in the fine-grained model implies fairness and nonwastefulness in the original extended model, and vice versa. To this end, as well as in Section 4, we show that HM-stability is equivalent to the stability defined in this section (Lemma 3 in Appendix A.3), and that $C h_{C}$ satisfies these properties (Lemma 4 in Appendix A.3). Then, we obtain the following theorem.

Theorem 4. A stable matching always exists, and DA-OT* is strategy-proof and obtains a stable matching, which is student-optimal among all the stable matchings.

Proof. Hatfield and Milgrom (2005) show that when $C h_{C}$ satisfies these three properties, a HM-stable matching always exists and the generalized DA mechanism is strategy-proof and obtains the student-optimal matching among all the HM-stable matchings. DA-OT* is one instance of the generalized DA mechanism. Also, $C h_{C}$ satisfies these properties (Lemma 4). Thus, DA-OT* is strategy-proof and obtains the student-optimal HM-stable matching. It 
is clear that a matching obtained by DA-OT* is valid. From Lemma 3, there exists oneto-one mapping between valid HM-stable matchings in the fine-grained model and stable matchings in the original extended model. Thus, DA-OT* obtains the student-optimal stable matching.

As in the basic model, DA-OT* has the time complexity of $O\left(|X|^{3}\right)$. We also have the two following propositions, which correspond to Theorem 2: one is for a case where the soft lower-bound for one particular type is increased; the other is for a case where the soft upper-bound for one particular type is increased. The proofs are omitted because that of Theorem 2 is straightforwardly applied.

Proposition 1. Consider DA-OT under $p_{C, T}$ and $q_{C, T}$ and under $p_{C, T}^{\prime}$ and $q_{C, T}$. Here $p_{C, T}$ and $p_{C, T}^{\prime}$ are almost identical, i.e., $p_{c, t}=p_{c, t}^{\prime}$ holds except for one particular school $c^{*}$ and one particular type $t^{*}$. We assume $p_{c^{*}, t^{*}}<p_{c^{*}, t^{*}}^{\prime}$ holds. For a given preference profile, let $X^{\prime}$ and $Y^{\prime}$ be the set of contracts obtained with $p_{C, T}$ and $p_{C, T}^{\prime}$, respectively. Then there exists at least one student $s$ where $t^{*} \in \tau(s)$ such that $Y_{s}^{\prime} \succeq_{s} X_{s}^{\prime}$.

Proposition 2. Consider DA-OT* under $p_{C, T}$ and $q_{C, T}$ and under $p_{C, T}$ and $q_{C, T}^{\prime}$. Here $q_{C, T}$ and $q_{C, T}^{\prime}$ are almost identical, i.e., $q_{c, t}=q_{c, t}^{\prime}$ holds except for one particular school $c^{*}$ and one particular type $t^{*}$. We assume $q_{c^{*}, t^{*}}<q_{c^{*}, t^{*}}^{\prime}$ holds. For a given preference profile, let $X^{\prime}$ and $Y^{\prime}$ be the set of contracts obtained with $q_{C, T}$ and $q_{C, T}^{\prime}$, respectively. Then there exists at least one student $s$ where $t^{*} \in \tau(s)$ such that $Y_{s}^{\prime} \succeq_{s} X_{s}^{\prime}$.

\section{Evaluation}

This section evaluates our newly developed DA-OT by computer simulation. As far as the authors are aware, DA-OT is the only known strategy-proof mechanism that is guaranteed to obtain a stable matching in our problem setting. More specifically, it can flexibly allocate seats across types, i.e., it is nonwasteful, and is fair across types, i.e., Condition (fr-i) in Definition 3 covers a case where $s$ and $s^{\prime}$ have different types.

In our evaluation, we use a benchmark mechanism called Artificial Cap Deferred Acceptance mechanism (ACDA). Although this mechanism does not obtain a stable matching, ${ }^{5}$ there are several reasons to use this mechanism as a benchmark: (1) it is basically identical to the basic DA mechanism (in which maximum quotas are defined artificially), which is a standard mechanism in matching theory, (2) this idea is used to satisfy several diversity constraints (Abdulkadiroğlu, 2005; Ergin \& Sönmez, 2006), (3) it is actually employed by Japan Residency Matching Program to handle distributional constraints (regional caps) (Kamada \& Kojima, 2015), and (4) it is used as a benchmark by Fragiadakis et al. (2016) and Goto, Iwasaki, Kawasaki, Kurata, Yasuda, and Yokoo (2016). More precisely, ACDA works as follows. We first fix the number of seats for each type, i.e., we assume school $c$ is divided into multiple sub-schools $c_{t_{1}}, c_{t_{2}}, \ldots$ with maximum quotas $\widetilde{q}_{c, t_{1}}, \widetilde{q}_{c, t_{2}}, \ldots$, respectively. Second, we set $\widetilde{q}_{c, t}$ such that $p_{c, t} \leq \widetilde{q}_{c, t}$ holds for all $c \in C, t \in T$, and $\sum_{t \in T} \widetilde{q}_{c, t}=q_{c}$ holds for all $c \in C$. Finally, we apply the standard DA mechanism. This mechanism is wasteful, but no student claims an empty seat by type. Also, it removes justified envy among the students with the same type defined in Condition (fr-i).

5. Unfortunately, there exists no other strategy-proof and stable mechanism in our setting besides DA-OT. 


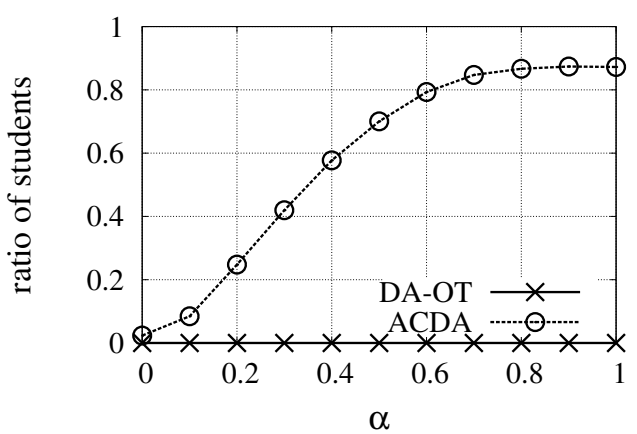

(a) Ratio of claiming students.

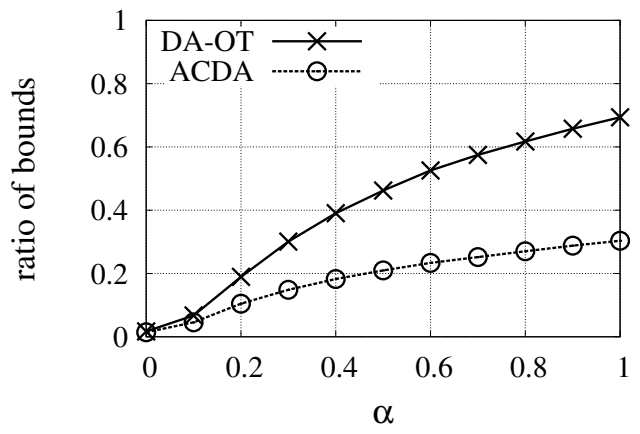

(c) Ratio of unfilled lower bounds.

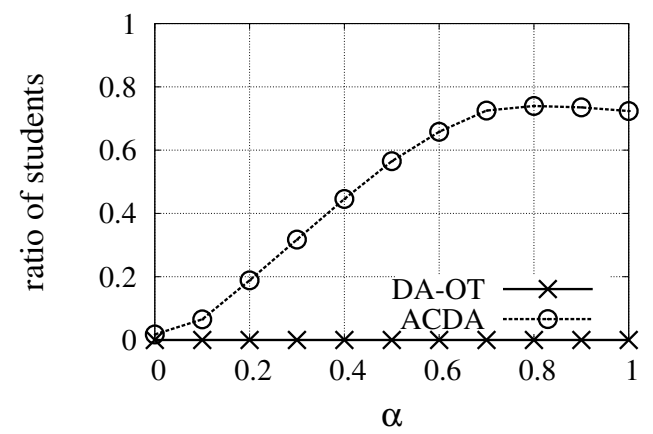

(b) Ratio of students with envy.

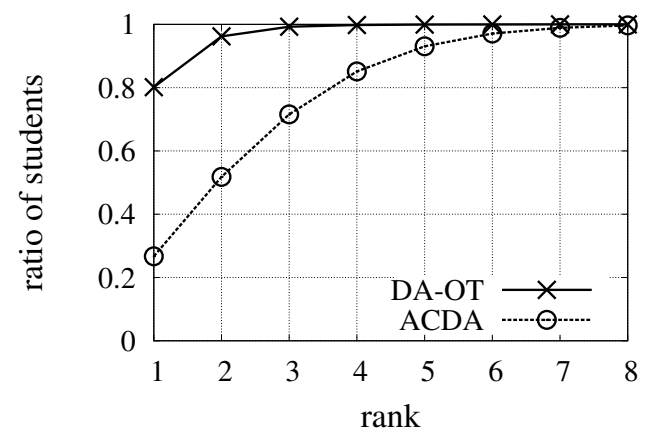

(d) CDFs of students' welfare $(\alpha=0.5)$.

Figure 1: Comparison between DA-OT and ACDA in the linear model $(n=256, m=8$, and $k=4)$.

We compare DA-OT and ACDA as follows. We consider a market with $n=256$ students, $m=8$ schools, and $k=\{2,4,6,8\}$ types. We mainly use $k=4$ unless otherwise noted. For each $c \in C$, we set $q_{c}$ to 48 , and for each type $t \in T$, we set $p_{c, t}$ to 4 . For each student $s \in S$, we set $|\tau(s)|$, i.e., the number of $s$ 's types, to 2 . For each student $s$, we randomly generate $\tau(s)$. Thus, for each pair of types $\left\{t_{i}, t_{j}\right\}$, the number of students whose types are $\left\{t_{i}, t_{j}\right\}$ is approximately $n /{ }_{k} C_{2}$. For ACDA, we set $\widetilde{q}_{c, t}$ to $q_{c} / k$ for each $c \in C$ and $t \in T$.

We generate students' preferences by utilizing following two models: (i) the linear model (Fragiadakis et al., 2016; Goto et al., 2014, 2016), and (ii) the Mallows model (Tubbs, 1992; Lu \& Boutilier, 2014; Drummond \& Boutilier, 2013b) ${ }^{6}$. In the linear model, we create one common $m \times k$ matrix $V^{*}$, where each element $v_{c, t}^{*}$ is uniformly drawn from [0,1] at random. Then for each student $s \in S$, we create private $m \times k$ matrix $V^{s}$, where each element $v_{c, t}^{s}$ is uniformly drawn from $[0,1]$ at random. Then we construct cardinal utilities over all $m$

6. We have also examined Prackett-Luce model (Perrault, Drummond, \& Bacchus, 2016) and confirmed that obtained results are qualitatively similar. 


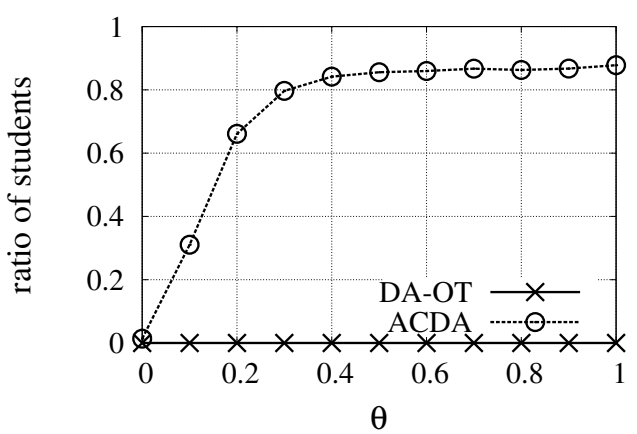

(a) Ratio of claiming students.

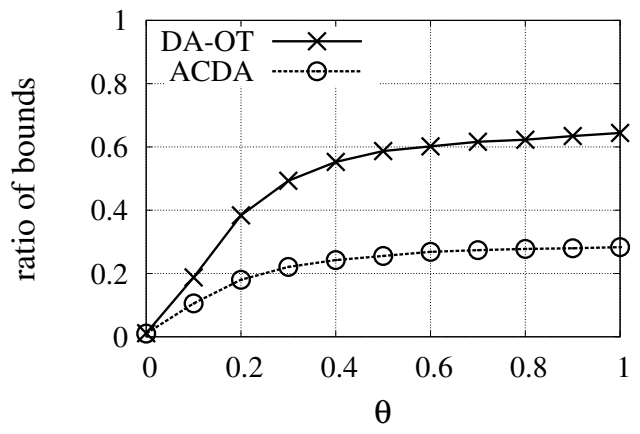

(c) Ratio of unfilled lower bounds.

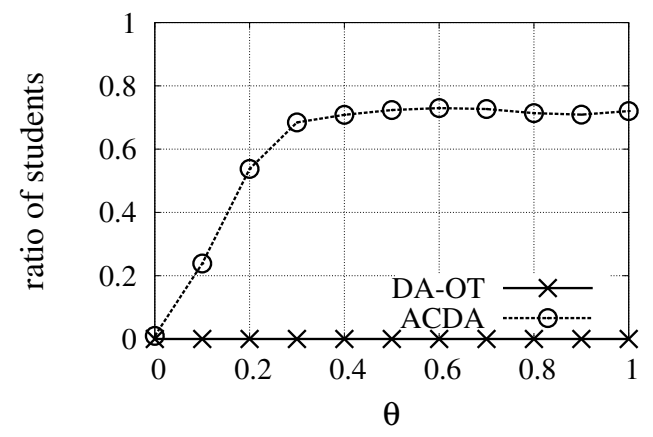

(b) Ratio of students with envy.

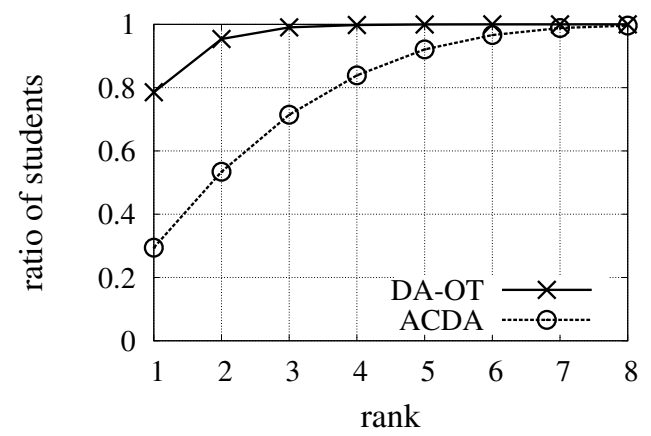

(d) CDFs of students' welfare $(\theta=0.2)$.

Figure 2: Comparison between DA-OT and ACDA in the Mallows model $(n=256, m=8$, and $k=4)$.

schools and $k$ types for student $s$ as $\alpha V^{*}+(1-\alpha) V^{s}$, for some $\alpha \in[0,1]$, i.e., the cardinal utility of contract $(s, c, t)$ is $\alpha v_{c, t}^{*}+(1-\alpha) v_{c, t}^{s}$. We then convert these cardinal utilities into an ordinal preference relation for each student. The higher the value of $\alpha$ is, the more correlated the students' preferences are. On the other hand, in the Mallows model, we draw the strict preference $\succ_{s}$ of a student $s$ from all possible $(m \times|\tau(s)|)$ ! preferences. The probability of choosing $\succ_{s}$ is expressed as

$$
\operatorname{Pr}\left(\succ_{s}\right)=\frac{\exp \left(-\theta d\left(\succ_{s}, \succ_{s}^{\prime}\right)\right)}{\sum_{\succ_{s}^{\prime \prime}} \exp \left(-\theta d\left(\succ_{s}^{\prime \prime}, \succ_{s}^{\prime}\right)\right)} .
$$

Here, $\theta \in \mathbb{R}$ denotes a spread parameter, $\succ_{s}^{\prime}$ is a central preference, which is randomly chosen from all possible preferences in our setting, and $d\left(\succ_{s}, \succ_{s}^{\prime}\right)$ represents the Kendall distance between $\succ_{s}$ and $\succ_{s}^{\prime}$. The distance is measured by the number of ordered pairs in $\succ_{s}$ that is inconsistent with those in $\succ_{s}^{\prime}$. When $\theta=0$, it becomes identical to the uniform distribution. 
Finally, the priority ranking of each school $c$ is drawn uniformly at random. We create 100 problem instances for each model and each parameter setting.

Figures 1a and $2 \mathrm{a}$ show the ratio of students who claim an empty seat. The x-axis denotes the value of $\alpha$ in the linear model, while it denotes the value of $\theta$ in the Mallows model. The y-axis denotes the average ratio of students who claim an empty seat. Since DA-OT is nonwasteful, no student claims an empty seat. We observe that both models provide a similar performances in the sense that students are more likely to claim an empty seat in ACDA as $\alpha$ or $\theta$ increases, e.g., more than $80 \%$ of students claim an empty seat when $\alpha \geq 0.6$ or $\theta \geq 0.4$. This is because as students' preferences become more correlated, the competition among the students becomes more severe. Also, the ratio is more sensitive to $\theta$ in the Mallows model than $\alpha$ in the linear model.

Figures $1 \mathrm{~b}$ and $2 \mathrm{~b}$ show the ratio of students with justified envy. The $\mathrm{x}$-axis is the same as in Figures 1a and 2a. Since DA-OT is fair, no student has justified envy. The qualitative trend is quite similar to those in Figures 1a and 2a. Students are more likely to have justified envy in ACDA as $\alpha$ or $\theta$ increases, e.g., more than $70 \%$ have justified envy when $\alpha \geq 0.7$ or $\theta \geq 0.3$.

Figures $1 \mathrm{c}$ and $2 \mathrm{c}$ show the ratio of unfilled lower bounds, i.e., we calculate the average of $\left(\sum_{c} \sum_{t} \max \left(p_{c, t}-\left|X_{c, t}^{\prime}\right|, 0\right)\right) / \sum_{c} \sum_{t} p_{c, t}$ for all $c$ and $t$. The $\mathrm{x}$-axis is the same as in Figures 1a and 2a. ACDA fills the soft lower bounds better than DA-OT and the difference increases as $\alpha$ or $\theta$ increases. For example, in the Mallows model, when $\theta=1$, ACDA fails to fill about $30 \%$ of the bounds, while DA-OT fails to fill about $60 \%$ of the bounds. In ACDA, the number of students of each type becomes similar across schools, since we artificially lower the maximum quotas. As a result, it is more likely to fill the bounds.

Figures $1 \mathrm{~d}$ and $2 \mathrm{~d}$ illustrate the students' welfare by plotting the cumulative distribution functions (CDFs) of the average number of students matched with their $i$-th or higher ranked contract when $\alpha$ is 0.5 (and $\theta$ is 0.2 ). A steep upper trend line is desirable. DA-OT performs much better than ACDA for all $\alpha$ or $\theta$. In Figure 1d, we can see, for example, $80 \%$ of students obtain their first choice, and $96 \%$ obtain their first or second choice in DA-OT. While in ACDA, only $27 \%$ of students obtain their most preferred contracts, and $52 \%$ obtain their most or second preferred contracts. We see a very similar trend for the Mallows model in Figure 2d. We can see that setting artificial caps decreases students' welfare, since it abandons too much flexibility.

In summary, DA-OT has clear advantage over ACDA in terms of fairness, nonwastefulness, and students' welfare. Although ACDA is better in filling lower bounds, it sacrifices the stability and students' welfare.

From now on, we concentrate on the linear model, since the obtained results with these two models are qualitatively similar. Figure 3 shows the results where the numbers of students and schools increased, i.e., we set the number of students $n$ to 512 , and the number of schools $m$ to 16 . For ACDA, we set $\widetilde{q}_{c, t}$ to $q_{c} / k=12$. We can see that the qualitative trends in this setting are quite similar to the previous setting. These results show that DA-OT has clear advantage over ACDA in terms of all criteria except the ratio of filled lower bounds.

Table 1 and Figure 4 examine the effect of $k=|T|$, i.e., the size of the type space. We fix $\alpha$ at 0.5 and vary $k$ from 2 to 8 by 2 . For ACDA, as $k$ increases, the number of students who claim empty seats or have justified envy increases, e.g., when $k=2$ and 8 , those who claims 


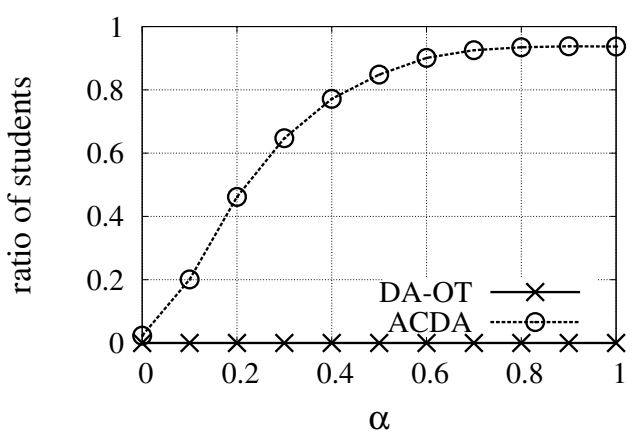

(a) Ratio of claiming students.

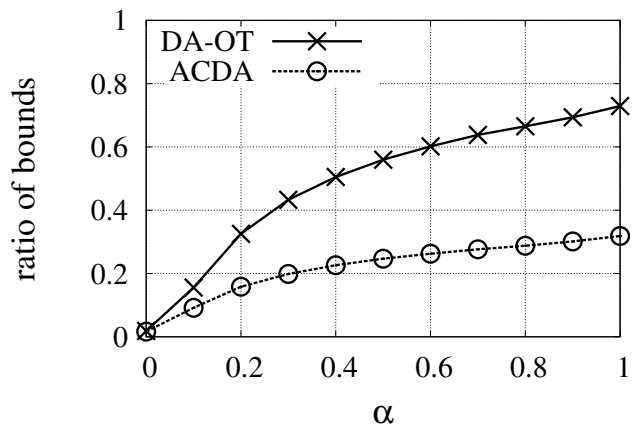

(c) Ratio of unfilled lower bounds.

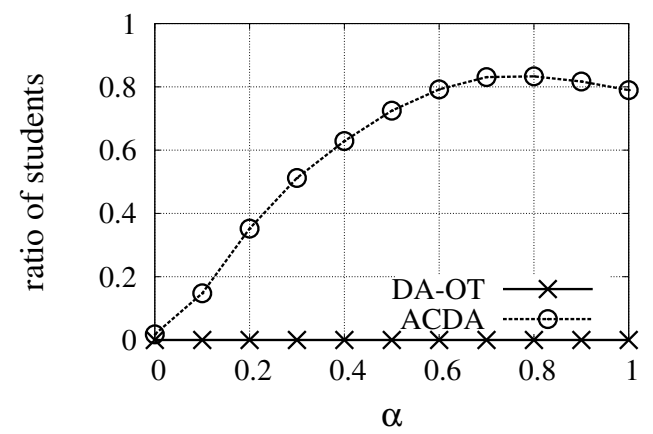

(b) Ratio of students with envy.

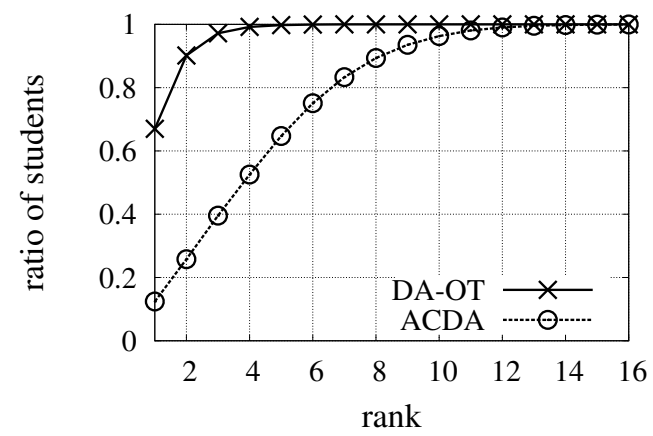

(d) CDFs of students' welfare $(\alpha=0.5)$.

Figure 3: Comparison between DA-OT and ACDA in the linear model $(n=512, m=16$, and $k=4)$.

an empty seat are 0.566 and 0.740 , respectively. As $k$ increases, the artificial maximum quota of each sub-school becomes smaller. As a result, the inflexibility of ACDA becomes more obvious.

For unfilled lower bounds, ACDA always outperforms DA-OT regardless of the size of the type. As $k$ increases, the ratio of unfilled lower bounds increases for both mechanisms. Since students are partitioned into smaller groups when $k$ is large (note that we fix the total number of students $n=256$ ), satisfying lower bounds for these groups becomes harder.

Figure 4 illustrates CDFs for DA-OT and ACDA for each $k$. While the CDFs of ACDA are almost the same, the CDFs of DA-OT improves as $k$ increases. Since students are partitioned into smaller groups when $k$ is large, they face less competitive situations. Thus, they are likely to be accepted to their preferred schools in DA-OT, which can assign students more flexibly than ACDA.

Next let us examine the case where type-specific ceilings are additionally imposed. We use almost the same setting as the comparison between DA-OT and ACDA when $n=256$ and $m=8$, except that we set $q_{c, t}$, i.e., the type-specific ceiling, to 16 for each school $c$ 
Table 1: Effect of type space size $k$ : fairness, nonwastefulness, and unfilled lower bounds $(n=256, m=8$, and $\alpha=0.5)$.

\begin{tabular}{|c||c|c|c|c|}
\hline Ratio & $k=2$ & 4 & 6 & 8 \\
\hline \hline Claiming students (ACDA) & 0.566 & 0.700 & 0.733 & 0.740 \\
\hline Students with envy (ACDA) & 0.449 & 0.565 & 0.593 & 0.595 \\
\hline Unfilled lower bounds (DA-OT) & 0.331 & 0.462 & 0.538 & 0.577 \\
\hline Unfilled lower bounds (ACDA) & 0.147 & 0.210 & 0.254 & 0.284 \\
\hline
\end{tabular}

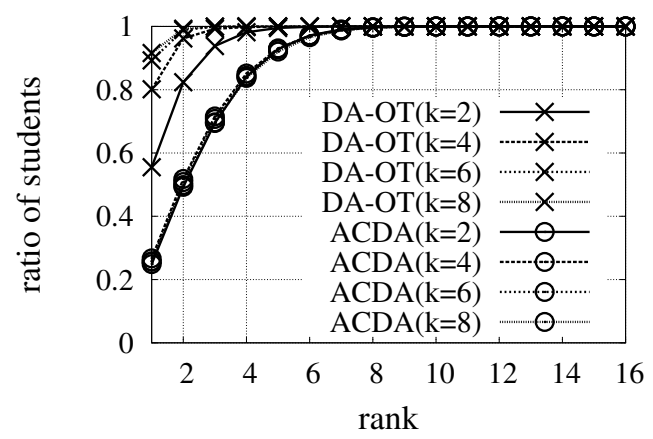

Figure 4: Effect of type space size $k$ : CDFs of students' welfare $(n=256, m=8$, and $\alpha=0.5)$.

and each type $t$. Here, we compare DA-OT* and DA-OT. If we apply DA-OT (which does not respect type-specific ceilings) in this setting, there is a chance that a student claims an empty seat by type. On the other hand, no student has justified envy or claims an empty seat, since DA-OT tries to allocate students based on each school's priority without considering type-specific ceilings. If we apply ACDA in this setting, the obtained results are identical to the previous setting without type-specific ceilings; for each school and for each type, ACDA never allocates students beyond its artificial cap $\widetilde{q}_{c, t}=12$, which is smaller than type-specific ceiling $q_{c, t}=16$.

Figure 5a shows the ratio of students who claim an empty seat by type (in the sense of Definition 9). The x- and y-axes are identical to Figure 1a. In DA-OT, at most $12 \%$ of students claim an empty seat by type. We also examine how much extent the lower and upper bounds are respected. Figures $5 \mathrm{~b}$ and $5 \mathrm{c}$ show the ratios of unfilled lower bounds and violated upper bounds. For the lower bounds, since both handle the lower bounds in the same way, almost no difference exists between the two mechanisms. For the upper bounds, as $\alpha$ increases, the ratio increases and reaches 0.48 for DA-OT and 0.45 for DA-OT* when $\alpha=1$. Though DA-OT* clearly violates less upper bounds than DA-OT, the difference between them is not so large.

Figure 5d illustrates the students' welfare as in Figure 1d. We fix $\alpha$ to 0.5. The performance of DA-OT* is almost identical to that of DA-OT. These results imply that DA$\mathrm{OT}^{*}$ achieve stability in the extended model without sacrificing students' welfare. Finally, Table 2 examines the effect of $k=|T|$, i.e., the size of the type space. For DA-OT, as $k$ 


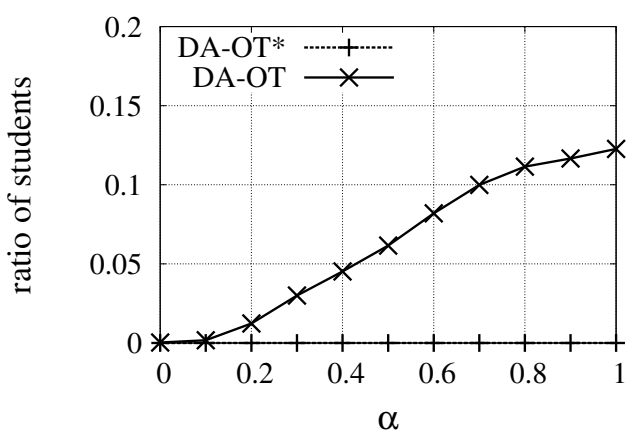

(a) Ratio of claiming students.

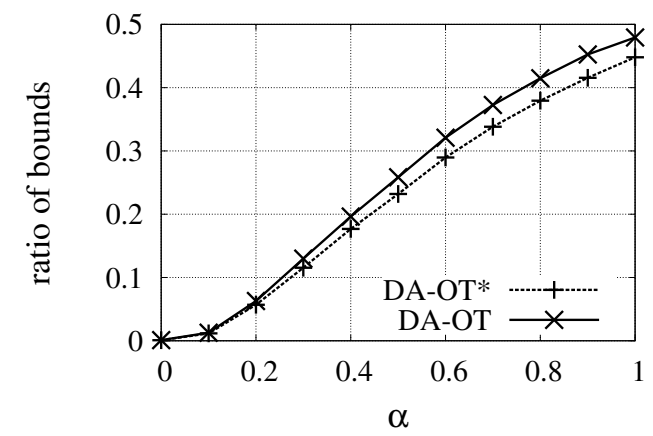

(c) Ratio of violated upper bounds.

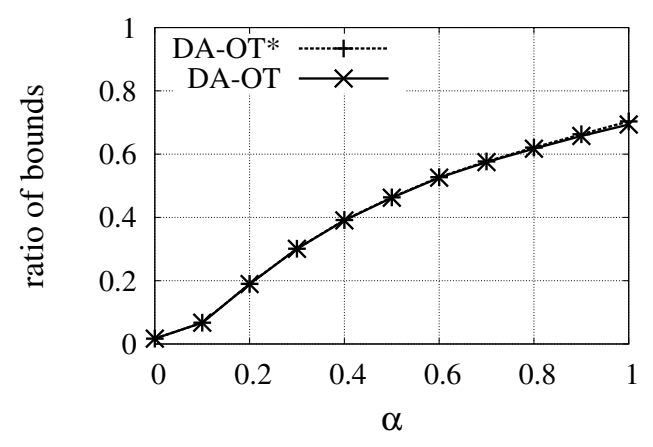

(b) Ratio of unfilled lower bounds.

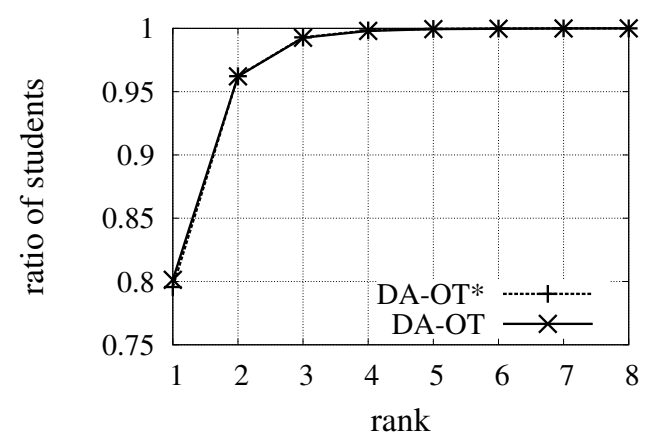

(d) CDFs of students' welfare $(\alpha=0.5)$.

Figure 5: Comparison between DA-OT and DA-OT ${ }^{*}$ in the linear model $(n=256, m=8$, and $k=4)$.

increases, the number of students who claims an empty seat by type decreases, e.g., when $k=2$ and 8 , the ratio are 0.198 and 0.018 , respectively. The ratios of unfilled lower bounds for DA-OT and DA-OT* are similar. Also, the ratios of unfilled lower bounds increase when $k$ becomes large. For the ratio of violated upper bounds, we calculate the average of $\left(\sum_{c} \sum_{t} \max \left(\left|X_{c, t}^{\prime}\right|-q_{c, t}, 0\right)\right) / \sum_{c} \sum_{t}\left|X_{c, t}^{\prime}\right|$. The ratios becomes smaller when $k$ increases. We omit the graph that illustrates students' welfare in this setting, since the effect of $k$ is almost negligible.

\section{Conclusion}

In this paper, we developed a new model for controlled school choice programs with softbounds, in which a student can belong to multiple types. We first presented a model that is a straightforward extension of an existing model for disjoint types. We proved that there exists a case where no matching is stable in this model and developed an alternative model. We showed that a stable matching is guaranteed to exist in this alternative model and 
Table 2: Effect of type space size $k$ : DA-OT and DA-OT ${ }^{*}(n=256, m=8$, and $\alpha=0.5)$.

\begin{tabular}{|c||c|c|c|c|}
\hline Ratio & $k=2$ & 4 & 6 & 8 \\
\hline \hline Claiming students (DA-OT) & 0.198 & 0.062 & 0.038 & 0.018 \\
\hline Unfilled lower bounds (DA-OT) & 0.331 & 0.462 & 0.538 & 0.577 \\
\hline Unfilled lower bounds (DA-OT*) & 0.334 & 0.464 & 0.539 & 0.577 \\
\hline Violated upper bounds (DA-OT) & 0.474 & 0.258 & 0.141 & 0.054 \\
\hline Violated upper bounds (DA-OT*) & 0.455 & 0.232 & 0.125 & 0.047 \\
\hline
\end{tabular}

developed the DA-OT mechanism, which is strategy-proof and obtains the student-optimal matching among all the stable matchings. Furthermore, we introduced an extended model that applies both type-specific ceilings and floors and developed DA-OT* mechanism that works in the extended model. We also showed computer simulation results, which illustrate that DA-OT outperforms ACDA (in which the number of seats for each type is fixed), and that DA-OT* achieves stability in the extended model without sacrificing students' welfare.

Our future works include developing a more general mechanism that can simultaneously handle different types of distributional constraints, e.g., regional minimum quotas (Goto et al., 2014).

\section{Acknowledgments}

This paper is based on the authors' conference publication (Kurata, Goto, Iwasaki, \& Yokoo, 2015). The main difference of this paper from it lies in Section 5; we therein introduce an extended model with a ceiling (in addition to a floor) that specifies a nonbinding target range, construct a mechanism for the extended model, and prove that it satisfies the same properties as the mechanism in Section 4. Furthermore, Section 6 is extended and includes the evaluation in larger-scale problem instances and an evaluation of the mechanism introduced in Section 5.

This work was partially supported by JSPS KAKENHI Grant Number 24220003 and 26280081. We appreciate Masahiro Goto for his helpful discussions and comments. We also highly appreciate the associated editor and three anonymous reviewers for their fruitful comments.

\section{Appendix A. Omitted Proofs}

In this section, we provide the omitted proofs.

\section{A.1 Lemmas for Theorem 1}

Lemma 1. Matching $X^{\prime}$ is HM-stable iff it is fair and nonwasteful.

Proof. We first show that HM-stability implies fairness and nonwastefulness. Assume $X^{\prime}$ is not fair, i.e., there exists $s, s^{\prime}$, where

$$
(s, c, t),\left(s^{\prime}, c^{\prime}, t^{\prime}\right) \in X^{\prime},\left(s, c^{\prime}, t^{\prime \prime}\right) \in X \backslash X^{\prime},\left(s, c^{\prime}, t^{\prime \prime}\right) \succ_{s}(s, c, t) \text {, and }\left(s, c^{\prime}, t^{\prime \prime}\right) \succ_{c^{\prime}}\left(s^{\prime}, c^{\prime}, t^{\prime}\right)
$$


hold. It is clear that $\left(s, c^{\prime}, t^{\prime \prime}\right) \in C h_{s}\left(X^{\prime} \cup\left\{\left(s, c^{\prime}, t^{\prime \prime}\right)\right\}\right)$ holds. Let us examine the choice function of school $c^{\prime}$. First, assume $t^{\prime}=t^{\prime \prime}$ (Condition (fr-i) in Definition 3) holds. Then $\left(s, c^{\prime}, t^{\prime}\right)$ is chosen before $\left(s^{\prime}, c^{\prime}, t^{\prime}\right)$ since $\left(s, c^{\prime}, t^{\prime}\right) \succ_{c^{\prime}}\left(s^{\prime}, c^{\prime}, t^{\prime}\right)$ holds. Therefore, $\left(s, c^{\prime}, t^{\prime}\right) \in$ $C h_{c^{\prime}}\left(X^{\prime} \cup\left\{\left(s, c^{\prime}, t^{\prime}\right)\right\}\right)$ holds. Thus $X^{\prime}$ is not HM-stable.

Second, assume $\left|X_{c^{\prime}, t^{\prime}}^{\prime}\right|>p_{c^{\prime}, t^{\prime}}$ (Condition (fr-ii) in Definition 3) holds. From the fact that $\left(s^{\prime}, c^{\prime}, t^{\prime}\right) \in C h_{c^{\prime}}\left(X^{\prime}\right),\left(s^{\prime}, c^{\prime}, t^{\prime}\right)$ is accepted at Step 3 in Definition 5, or there exists another contract $\left(s^{\prime \prime}, c^{\prime}, t^{\prime}\right)$ that is accepted at Step 3 in Definition 5 and $\left(s^{\prime}, c^{\prime}, t^{\prime}\right) \succ_{c^{\prime}}$ $\left(s^{\prime \prime}, c^{\prime}, t^{\prime}\right)$. When calculating $C h_{c^{\prime}}\left(X^{\prime} \cup\left\{\left(s, c^{\prime}, t^{\prime \prime}\right)\right\}\right)$, if $\left(s, c^{\prime}, t^{\prime \prime}\right)$ is not accepted at Step 2, it should be accepted at Step 3, since $\left(s, c^{\prime}, t^{\prime \prime}\right)$ is chosen before $\left(s^{\prime}, c^{\prime}, t^{\prime}\right)$ or $\left(s^{\prime \prime}, c^{\prime}, t^{\prime}\right)$. Thus, $\left(s, c^{\prime}, t^{\prime \prime}\right) \in C h_{c^{\prime}}\left(X^{\prime} \cup\left\{\left(s, c^{\prime}, t^{\prime \prime}\right)\right\}\right)$ holds; $X^{\prime}$ is not HM-stable.

Third, assume $s$ claims an empty seat of $c^{\prime}$. Thus $(s, c, t) \in X^{\prime},\left(s, c^{\prime}, t^{\prime}\right) \in X \backslash X^{\prime}$, and $\left(s, c^{\prime}, t^{\prime}\right) \succ_{s}(s, c, t)$ hold. It is clear that $\left(s, c^{\prime}, t^{\prime}\right) \in C h_{s}\left(X^{\prime} \cup\left\{\left(s, c^{\prime}, t^{\prime}\right)\right\}\right)$. Also, if $\left|X_{c^{\prime}}\right|<q_{c^{\prime}}$, i.e., Condition (nw-i) in Definition 4, holds, it is clear that $\left(s, c^{\prime}, t^{\prime}\right) \in C h_{c^{\prime}}\left(X^{\prime} \cup\left\{\left(s, c^{\prime}, t^{\prime}\right)\right\}\right)$. Furthermore, if $c=c^{\prime},\left(s, c, t^{\prime}\right) \succ_{c}(s, c, t)$, i.e., Condition (nw-ii) in Definition 4, holds, then either $(s, c, t)$ is accepted at Step 3 in Definition 5 , or there exists another contract $\left(s^{\prime}, c, t\right)$ that is accepted at Step 3 in Definition 5 and $(s, c, t) \succ_{c}\left(s^{\prime}, c, t\right)$. Thus, when calculating $C h_{c}\left(X^{\prime} \cup\left\{\left(s, c, t^{\prime}\right)\right\}\right)$, if $\left(s, c, t^{\prime}\right)$ is not accepted at Step 2, it should be accepted at Step 3 since $\left(s, c, t^{\prime}\right)$ is chosen before $(s, c, t)$ or $\left(s^{\prime}, c, t\right)$. Thus, $\left(s, c, t^{\prime}\right) \in C h_{c}\left(X^{\prime} \cup\left\{\left(s, c, t^{\prime}\right)\right\}\right)$ holds; $X^{\prime}$ is not HM-stable.

Finally, assume $s$ claims an empty seat of $c^{\prime}$ by type. Thus, $\left(s, c^{\prime}, t^{\prime}\right) \in X \backslash X^{\prime}$ and $\left(s, c^{\prime}, t^{\prime}\right) \succ_{s}(s, c, t)$ holds. It is clear that $\left(s, c^{\prime}, t^{\prime}\right) \in C h_{s}\left(X^{\prime} \cup\left\{\left(s, c^{\prime}, t^{\prime}\right)\right\}\right)$. Also, $\left|X_{c^{\prime}, t^{\prime}}\right|<$ $p_{c^{\prime}, t^{\prime}}$, i.e., Condition (nw-iii) in Definition 4, holds. Then, $\left(s, c^{\prime}, t^{\prime}\right) \in C h_{c^{\prime}}\left(X^{\prime} \cup\left\{\left(s, c^{\prime}, t^{\prime}\right)\right\}\right)$ holds since $\left(s, c^{\prime}, t^{\prime}\right)$ should be accepted at Step 2; $X^{\prime}$ is not HM-stable.

Next, let us show that fairness and nonwastefulness imply HM-stability. Assume matching $X^{\prime}$ is not HM-stable, i.e., there exists $(s, c, t) \in X \backslash X^{\prime}$ such that $(s, c, t) \in C h_{s}\left(X^{\prime} \cup\right.$ $\{(s, c, t)\})$ and $(s, c, t) \in C h_{c}\left(X^{\prime} \cup\{(s, c, t)\}\right)$ hold. Assume $\left(s, c^{\prime}, t^{\prime}\right) \in X^{\prime}$. It is clear that $(s, c, t) \succ_{s}\left(s, c^{\prime}, t^{\prime}\right)$ holds since $(s, c, t) \in C h_{s}\left(X^{\prime} \cup\{(s, c, t)\}\right)$.

Assume $C h_{c}\left(X^{\prime} \cup\{(s, c, t)\}\right)=C h_{c}\left(X^{\prime}\right) \cup\{(s, c, t)\}$, i.e., no contract is rejected as the consequence of accepting $(s, c, t)$. Then $\left|X_{c}^{\prime}\right|<q_{c}$ must hold and $X^{\prime}$ is wasteful from Condition (nw-i) in Definition 4. Thus, let us assume $\left(s^{\prime}, c, t^{\prime \prime}\right) \in C h_{c}\left(X^{\prime}\right)$ and $\left(s^{\prime}, c, t^{\prime \prime}\right) \notin$ $C h_{c}\left(X^{\prime} \cup\{(s, c, t)\}\right)$ hold, i.e., $\left(s^{\prime}, c, t^{\prime \prime}\right)$ is rejected as the consequence of accepting $(s, c, t)$.

First, consider the case where $\left|X_{c, t}^{\prime}\right|<p_{c, t}$. Then, $s$ claims an empty seat of $c$ by type since Condition (nw-iii) in Definition 4 holds. Next, consider the case where $s=s^{\prime}$ (and $\left.t^{\prime \prime}=t^{\prime}\right)$. Then $(s, c, t) \succ_{c}\left(s, c, t^{\prime}\right)$ and $\left|X_{c, t^{\prime}}^{\prime}\right|>p_{c, t^{\prime}}$ holds. Thus, $s$ claims an empty seat of $c$ since Condition (nw-ii) in Definition 4 holds. Finally, consider the case where $s \neq s^{\prime}$. Then either (i) $t=t^{\prime \prime}$ and $(s, c, t) \succ_{c}\left(s^{\prime}, c, t\right)$, or (ii) $(s, c, t) \succ_{c}\left(s^{\prime}, c, t^{\prime \prime}\right)$ and $\left|X_{c, t^{\prime \prime}}^{\prime}\right|>p_{c, t^{\prime \prime}}$ holds. In case (i) $s$ has justified envy toward $s^{\prime}$ since $(s, c, t) \succ_{c}\left(s^{\prime}, c, t\right)$, and Condition (fr-i) in Definition 3 holds. In case (ii), $s$ has justified envy toward $s^{\prime}$ since $(s, c, t) \succ_{c}\left(s^{\prime}, c, t\right)$, and Condition (fr-ii) in Definition 3 holds.

Lemma 2. $C h_{C}$ satisfies the following properties.

Irrelevance of rejected contracts: For any $X^{\prime} \subseteq X$ and $x \in X \backslash X^{\prime}, C h_{C}\left(X^{\prime}\right)=C h_{C}\left(X^{\prime} \cup\right.$ $\{x\})$ if $x \notin C h_{C}\left(X^{\prime} \cup\{x\}\right)$.

Law of aggregate demand: For any $X^{\prime}, X^{\prime \prime} \subseteq X$ with $X^{\prime} \subseteq X^{\prime \prime},\left|C h_{C}\left(X^{\prime}\right)\right| \leq\left|C h_{C}\left(X^{\prime \prime}\right)\right|$. 
Substitutes condition: For any $X^{\prime}, X^{\prime \prime} \subseteq X$ with $X^{\prime} \subseteq X^{\prime \prime}, X^{\prime} \backslash C h_{C}\left(X^{\prime}\right) \subseteq X^{\prime \prime} \backslash C h_{C}\left(X^{\prime \prime}\right)$.

Proof. It is obvious that the irrelevance of rejected contracts holds from Definition 5. To show the law of aggregate demand and the substitutes condition, it is sufficient to show that each individual choice function $C h_{c}$ satisfies the following conditions:

(a) For any $X^{\prime} \subseteq X$ and $x \in X \backslash X^{\prime},\left|C h_{c}\left(X^{\prime}\right)\right| \leq\left|C h_{c}\left(X^{\prime} \cup\{x\}\right)\right|$.

(b) For any $X^{\prime} \subseteq X$ and $x \in X \backslash X^{\prime}, X^{\prime} \backslash C h_{c}\left(X^{\prime}\right) \subseteq\left(X^{\prime} \cup\{x\}\right) \backslash C h_{c}\left(X^{\prime} \cup\{x\}\right)$.

From Definition 5, it is clear that if $\left|X_{c}^{\prime}\right| \leq q_{c}$, then $C h_{c}\left(X^{\prime}\right)=X^{\prime}$ holds. Also, if $\left|X_{c}^{\prime}\right| \geq q_{c}$, then $\left|C h_{c}\left(X^{\prime}\right)\right|=q_{c}$ holds. In words, if the number of contracts related to $c$ is less than or equal to $q_{c}$, then no contract is rejected. Also, if the number of contracts related to $c$ is more than or equal to $q_{c}$, then the number of accepted contracts equals $q_{c}$. Thus, it is clear that condition (a) holds.

Assume condition (b) is not satisfied, i.e., there exists $X^{\prime} \subseteq X, x \in X \backslash X^{\prime}, x^{\prime} \in$ $X^{\prime} \backslash C h_{c}\left(X^{\prime}\right)$, such that $x^{\prime} \in C h_{c}\left(X^{\prime} \cup\{x\}\right)$, i.e., $x^{\prime}$ is rejected from $c$ when $X^{\prime}$ is present, but it is accepted when $X^{\prime} \cup\{x\}$ is present. There can be multiple contracts that satisfy the above conditions. Without loss of generality, we assume $x^{\prime}$ has the highest priority ranking among such contracts. It is clear that $x$ must be a contract related to $c$ and $x \in C h_{c}\left(X^{\prime} \cup\{x\}\right)$. If $\left|X_{c}^{\prime}\right| \leq q_{c}$, no contract is rejected, and thus $\left|X_{c}^{\prime}\right|>q_{c}$ holds. Furthermore, $\left|C h_{c}\left(X^{\prime}\right)\right|=\left|C h_{c}\left(X^{\prime} \cup\{x\}\right)\right|=q_{c}$ holds. When calculating $C h_{c}\left(X^{\prime} \cup\{x\}\right)$, there are four possibilities: (i) both $x$ and $x^{\prime}$ are accepted at Step 2 in Definition 5, (ii) $x$ is accepted at Step 2 while $x^{\prime}$ is accepted at Step 3, (iii) $x$ is accepted at Step 3 while $x^{\prime}$ is accepted at Step 2, or (iv) both are accepted at Step 3.

In cases (i) or (iii), when $X^{\prime} \cup\{x\}$ is present, $x^{\prime}=\left(s, c, t^{\prime}\right)$ is accepted at Step 2 since $\left|Y_{c, t^{\prime}}\right|<p_{c, t^{\prime}}$ holds. Then, when only $X^{\prime}$ is present, $x^{\prime}$ must be chosen before $\left|Y_{c, t^{\prime}}\right|$ becomes equal to $p_{c, t^{\prime}}$. Thus, $x^{\prime}$ must be accepted also in this situation. This is a contradiction. Thus, cases (i) and (iii) are not possible. Also, in cases (ii) or (iv), when $x^{\prime}$ is accepted at Step 3 when $X^{\prime} \cup\{x\}$ is present, $|Y|<q_{c}$ holds. When only $X^{\prime}$ is present, $x^{\prime}$ must be chosen before $|Y|$ becomes equal to $q_{c}$. Thus, $x^{\prime}$ must be accepted also in this situation. This is a contradiction. Thus, cases (ii) and (iv) are not possible.

\section{A.2 Proof of Theorem 2}

Proof. Hatfield and Kojima (2009) show that if a mechanism always produces the studentoptimal stable matching, the mechanism is weakly group strategy-proof, i.e., no group of students can make each of its members strictly better off by jointly misreporting their preferences. Since DA-OT always produces the student-optimal stable matching, it is weakly group strategy-proof. We are going to show that if we assume the claim of Theorem 2 does not hold, then it violates the fact that the DA-OT is weakly group strategy-proof.

Let us assume the preference profile of students is $\succ_{S}$. When the vector of soft-bounds is $p_{C, T}$, the obtained matching is $X^{\prime}$. When the vector of soft-bounds is changed to $p_{C, T}^{\prime}$, where $p_{c^{*}, t^{*}}<p_{c^{*}, t^{*}}^{\prime}$ holds for one particular school $c^{*}$ and one particular type $t^{*}$, the obtained matching is $Y^{\prime}$. Let $S^{\prime}$ be the set of students $\left\{s \in S \mid t^{*} \in \tau(s)\right\}$. Assume by way of contradiction, for all $s \in S^{\prime}, X_{s}^{\prime} \succ_{s} Y_{s}^{\prime}$ holds. 
Let us consider a situation where $S^{\prime}$ jointly modify their preferences such that each $s \in S^{\prime}$ lists $X_{s}^{\prime}$ as her top choice. Let $\succ_{s}^{\prime}$ be this preference. Also, for each $s \in S \backslash S^{\prime}$, her preference does not change. Thus, let $\succ_{S}^{\prime}$ be the preference profile $\left(\left(\succ_{s}^{\prime}\right)_{s \in S^{\prime}},\left(\succ_{s}\right)_{s \in S \backslash S^{\prime}}\right)$.

We first show that $X^{\prime}$ is a HM-stable matching under $p_{C, T}$ and $\succ_{S}^{\prime}$. Since each $s \in S^{\prime}$ is getting her top choice in $X^{\prime}$, none of them has a justified envy or claims an empty seat. Therefore, $X^{\prime}$ is a HM-stable matching under $p_{C, T}$ according to $\succ_{S}^{\prime}$.

Next, we show that $X^{\prime}$ is a HM-stable matching under $p_{C, T}^{\prime}$ and $\succ_{S}^{\prime}$. Let us check each condition in Definitions 3 and 4. First, Conditions (fr-i) and (nw-i) are not affected by soft-bounds. Thus, when Conditions (fr-i) and (nw-i) do not hold under $p_{C, T}$ for any student, they do not hold under $p_{C, T}^{\prime}$ as well. Next, Conditions (fr-ii) and (nw-ii) are affected by soft-bounds, but these conditions become harder (i.e., less likely to hold) when any soft-bound increases. Thus, when Conditions (fr-ii) and (nw-ii) do not hold under $p_{C, T}$ for any student, they do not hold under $p_{C, T}^{\prime}$ as well. Third, Condition (nw-iii) is affected by soft-bounds. Let us assume $c^{\prime}=c^{*}$ and $t^{\prime}=t^{*}$ in the definition of (nw-iii). When the soft lower bound for type $t^{\prime}$ of school $c^{\prime}$ increases, student $s$ where $t^{\prime} \in \tau(s)$ is more likely to claim an empty seat. However, since student $s$ is assigned to her top choice in $X^{\prime}$ according $\succ_{S}^{\prime}$, she does not prefer any contract $\left(s, c^{\prime}, t^{\prime}\right)$ over $X_{s}^{\prime}$. Thus, no student claims an empty seat by type in $X^{\prime}$. As a result, as long as $X^{\prime}$ is HM-stable under $p_{C, T}$, it is HM-stable under $p_{C, T}^{\prime}$ as well.

Finally, let $Z^{\prime}$ be the resulting matching obtained by DA-OT under $p_{C, T}^{\prime}$ according to $\succ_{S}^{\prime}$. Since $Z^{\prime}$ is student-optimal among all HM-stable matchings under $\succ_{S}^{\prime}$, each student must obtain a contract that is at least as good as the contract she obtains in another HMstable matching. Here, for each student $s \in S^{\prime}$, the only contract that is as good as $X_{s}^{\prime}$ according to $\succ_{s}^{\prime}$ is $X_{s}^{\prime}$. Hence, all students in $S^{\prime}$ get a strictly better outcome by jointly misreporting their preferences, which contradicts the fact that DA-OT is weakly group strategy-proof.

\section{A.3 Lemmas for Theorem 4}

Lemma 3. Assume $X^{\prime}$ in the original model corresponds to $\tilde{X}^{\prime}$ in the extended model and vice versa. Then, $\tilde{X}^{\prime}$ is HM-stable iff $X^{\prime}$ is stable.

Proof. Since $X^{\prime}$ corresponds to $\tilde{X}^{\prime}, \tilde{X}^{\prime}$ is valid. We first show that HM-stability implies fairness and nonwastefulness. First, let us assume $s$ has justified envy toward $s^{\prime}$ by Condition (fr-i) in Definition 8, i.e., there exist $s$ and $s^{\prime}$, where

$$
(s, c, t),\left(s^{\prime}, c^{\prime}, t^{\prime}\right) \in X^{\prime},\left(s, c^{\prime}, t^{\prime}\right) \in X \backslash X^{\prime},\left(s, c^{\prime}, t^{\prime}\right) \succ_{s}(s, c, t) \text {, and }\left(s, c^{\prime}, t^{\prime}\right) \succ_{c^{\prime}}\left(s^{\prime}, c^{\prime}, t^{\prime}\right)
$$

hold. Then, in the fine-grained model,

$$
\begin{aligned}
& (s, c, t, k),\left(s^{\prime}, c^{\prime}, t^{\prime}, k^{\prime}\right) \in \tilde{X}^{\prime},\left(s, c^{\prime}, t^{\prime}, k^{\prime}\right) \in \tilde{X} \backslash \tilde{X}^{\prime}, \\
& \left(s, c^{\prime}, t^{\prime}, k^{\prime}\right) \tilde{\succ}_{s}(s, c, t, k), \text { and }\left(s, c^{\prime}, t^{\prime}, k^{\prime}\right) \tilde{\succ}_{c^{\prime}}\left(s^{\prime}, c^{\prime}, t^{\prime}, k^{\prime}\right)
\end{aligned}
$$

hold for some $k$ and $k^{\prime}$. It is clear that $\left(s, c^{\prime}, t^{\prime}, k^{\prime}\right) \in C h_{s}\left(\tilde{X}^{\prime} \cup\left\{\left(s, c^{\prime}, t^{\prime}, k^{\prime}\right)\right\}\right)$ holds. Also, since $\left(s, c^{\prime}, t^{\prime}, k^{\prime}\right)$ is examined before $\left(s^{\prime}, c^{\prime}, t^{\prime}, k^{\prime}\right)$ in calculating $C h_{c^{\prime}}\left(\tilde{X}^{\prime} \cup\left\{\left(s, c^{\prime}, t^{\prime}, k^{\prime}\right)\right\}\right)$, $\left(s, c^{\prime}, t^{\prime}, k^{\prime}\right) \in C h_{c^{\prime}}\left(\tilde{X}^{\prime} \cup\left\{\left(s, c^{\prime}, t^{\prime}, k^{\prime}\right)\right\}\right)$ holds. Thus, $\tilde{X}^{\prime}$ is not HM-stable. 
Next, assume $s$ has justified envy toward $s^{\prime}$ by Condition (fr-ii) in Definition 8, i.e., there exists $s, s^{\prime}$, where

$$
\begin{aligned}
& (s, c, t),\left(s^{\prime}, c^{\prime}, t^{\prime}\right) \in X^{\prime},\left(s, c^{\prime}, t^{\prime \prime}\right) \in X \backslash X^{\prime},\left(s, c^{\prime}, t^{\prime \prime}\right) \succ_{s}(s, c, t),\left(s, c^{\prime}, t^{\prime \prime}\right) \succ_{c^{\prime}}\left(s^{\prime}, c^{\prime}, t^{\prime}\right), \\
& \left|X_{c^{\prime}, t^{\prime}}^{\prime}\right|>p_{c^{\prime}, t^{\prime}}, \text { and }\left|X_{c^{\prime}, t^{\prime \prime}}^{\prime}\right|<q_{c^{\prime}, t^{\prime \prime}}
\end{aligned}
$$

hold. Then in the fine-grained model, $(s, c, t, k) \in \tilde{X}^{\prime}$ holds for some $k$ and there exists a contract $\left(s^{\prime \prime}, c^{\prime}, t^{\prime}, k^{\prime}\right) \in \tilde{X}^{\prime}$ such that $k^{\prime} \in\{2,3\},\left(s^{\prime}, c^{\prime}, t^{\prime}, 2\right) \tilde{\succ}_{c^{\prime}}\left(s^{\prime \prime}, c^{\prime}, t^{\prime}, k^{\prime}\right)$ or $s^{\prime}=s^{\prime \prime}$ holds. Then $\left(s, c^{\prime}, t^{\prime \prime}, 2\right) \tilde{\succ}_{c^{\prime}}\left(s^{\prime \prime}, c^{\prime}, t^{\prime}, k^{\prime}\right)$, and $\left(s, c^{\prime}, t^{\prime \prime}, 2\right) \tilde{\succ}_{s}(s, c, t, k)$ hold. It is clear that $\left(s, c^{\prime}, t^{\prime \prime}, 2\right) \in C h_{s}\left(\tilde{X}^{\prime} \cup\left\{\left(s, c^{\prime}, t^{\prime \prime}, 2\right)\right\}\right)$ holds. Also, since $\left(s, c^{\prime}, t^{\prime \prime}, 2\right)$ is examined before $\left(s^{\prime \prime}, c^{\prime}, t^{\prime}, k^{\prime}\right)$ in calculating $C h_{c^{\prime}}\left(\tilde{X}^{\prime} \cup\left\{\left(s, c^{\prime}, t^{\prime \prime}, 2\right)\right\}\right),\left(s, c^{\prime}, t^{\prime \prime}, 2\right) \in C h_{c^{\prime}}\left(\tilde{X}^{\prime} \cup\left\{\left(s, c^{\prime}, t^{\prime \prime}, 2\right)\right\}\right)$ holds. Thus $\tilde{X}^{\prime}$ is not HM-stable.

Next, assume $s$ has justified envy toward $s^{\prime}$ by Condition (fr-iii) in Definition 8, i.e., there exists $s, s^{\prime}$, where

$$
\begin{aligned}
& (s, c, t),\left(s^{\prime}, c^{\prime}, t^{\prime}\right) \in X^{\prime},\left(s, c^{\prime}, t^{\prime \prime}\right) \in X \backslash X^{\prime},\left(s, c^{\prime}, t^{\prime \prime}\right) \succ_{s}(s, c, t), \\
& \left(s, c^{\prime}, t^{\prime \prime}\right) \succ_{c^{\prime}}\left(s^{\prime}, c^{\prime}, t^{\prime}\right),\left|X_{c^{\prime}, t^{\prime}}^{\prime}\right|>q_{c^{\prime}, t^{\prime}}, \text { and }\left|X_{c^{\prime}, t^{\prime \prime}}^{\prime}\right| \geq q_{c^{\prime}, t^{\prime \prime}}
\end{aligned}
$$

hold. Then in the fine-grained model, $(s, c, t, k) \in \tilde{X}^{\prime}$ holds for some $k$ and there exists a contract $\left(s^{\prime \prime}, c^{\prime}, t^{\prime}, 3\right) \in \tilde{X}^{\prime}$ such that $\left(s^{\prime}, c^{\prime}, t^{\prime}, 3\right) \tilde{\succ}_{c^{\prime}}\left(s^{\prime \prime}, c^{\prime}, t^{\prime}, 3\right)$ or $s^{\prime}=s^{\prime \prime}$ holds. Then $\left(s, c^{\prime}, t^{\prime \prime}, 3\right) \tilde{\succ}_{c^{\prime}}\left(s^{\prime \prime}, c^{\prime}, t^{\prime}, 3\right)$, and $\left(s, c^{\prime}, t^{\prime \prime}, 3\right) \tilde{\succ}_{s}(s, c, t, k)$ hold. It is clear that $\left(s, c^{\prime}, t^{\prime \prime}, 3\right) \in$ $C h_{s}\left(\tilde{X}^{\prime} \cup\left\{\left(s, c^{\prime}, t^{\prime \prime}, 3\right)\right\}\right)$ holds. Also, since $\left(s, c^{\prime}, t^{\prime \prime}, 3\right)$ is examined before $\left(s^{\prime \prime}, c^{\prime}, t^{\prime}, 3\right)$ in calculating $C h_{c^{\prime}}\left(\tilde{X}^{\prime} \cup\left\{\left(s, c^{\prime}, t^{\prime \prime}, 3\right)\right\}\right),\left(s, c^{\prime}, t^{\prime \prime}, 3\right) \in C h_{c^{\prime}}\left(\tilde{X}^{\prime} \cup\left\{\left(s, c^{\prime}, t^{\prime \prime}, 3\right)\right\}\right)$ holds. Thus, $\tilde{X}^{\prime}$ is not HM-stable.

Next, assume $s$ claims an empty seat of $c^{\prime}$ by Condition (nw-i) in Definition 9, i.e.,

$$
(s, c, t) \in X^{\prime},\left(s, c^{\prime}, t^{\prime}\right) \in X \backslash X^{\prime},\left(s, c^{\prime}, t^{\prime}\right) \succ_{s}(s, c, t), \text { and }\left|X_{c^{\prime}}^{\prime}\right|<q_{c^{\prime}}
$$

hold. Then, in the fine-grained model,

$$
(s, c, t, k) \in \tilde{X}^{\prime},\left(s, c^{\prime}, t^{\prime}, 3\right) \in \tilde{X} \backslash \tilde{X}^{\prime},\left(s, c^{\prime}, t^{\prime}, 3\right) \tilde{\succ}_{s}(s, c, t, k) \text {, and }\left|\tilde{X}_{c^{\prime}}\right|<q_{c^{\prime}}
$$

hold for some $k$. It is clear that $\left(s, c^{\prime}, t^{\prime}, 3\right) \in C h_{s}\left(\tilde{X}^{\prime} \cup\left\{\left(s, c^{\prime}, t^{\prime}, 3\right)\right\}\right)$ and $\left(s, c^{\prime}, t^{\prime}, 3\right) \in$ $C h_{c^{\prime}}\left(\tilde{X}^{\prime} \cup\left\{\left(s, c^{\prime}, t^{\prime}, 3\right)\right\}\right)$ hold. Thus, $\tilde{X}^{\prime}$ is not HM-stable.

Next, assume $s$ claims an empty seat of $c$ by Condition (nw-ii) in Definition 9, i.e.,

$$
\begin{aligned}
& (s, c, t) \in X^{\prime},\left(s, c, t^{\prime}\right) \in X \backslash X^{\prime},\left(s, c, t^{\prime}\right) \succ_{s}(s, c, t),\left(s, c, t^{\prime}\right) \succ_{c}(s, c, t), \\
& \left|X_{c, t}^{\prime}\right|>p_{c, t}, \text { and }\left|X_{c, t^{\prime}}^{\prime}\right|<q_{c, t^{\prime}}
\end{aligned}
$$

hold. Then in the fine-grained model, there exists a contract $\left(s^{\prime}, c, t, k^{\prime}\right) \in \tilde{X}^{\prime}$ such that $k^{\prime} \in\{2,3\},(s, c, t, 2) \tilde{\succ}_{c}\left(s^{\prime}, c, t, k^{\prime}\right)$ or $s^{\prime}=s$ holds. Thus $\left(s, c, t^{\prime}, 2\right) \tilde{\succ}_{c}\left(s^{\prime}, c, t, k^{\prime}\right)$ holds. Also,

$$
(s, c, t, k) \in \tilde{X}^{\prime},\left(s, c, t^{\prime}, 2\right) \in \tilde{X} \backslash \tilde{X}^{\prime} \text {, and }\left(s, c, t^{\prime}, 2\right) \tilde{\succ}_{s}(s, c, t, k)
$$

hold for some $k$. It is clear that $\left(s, c, t^{\prime}, 2\right) \in C h_{s}\left(\tilde{X}^{\prime} \cup\left\{\left(s, c, t^{\prime}, 2\right)\right\}\right)$ holds. Also, since $\left(s, c, t^{\prime}, 2\right)$ is examined before $\left(s^{\prime}, c, t, k^{\prime}\right)$ when calculating $C h_{c}\left(\tilde{X}^{\prime} \cup\left\{\left(s, c, t^{\prime}, 2\right)\right\}\right),\left(s, c, t^{\prime}, 2\right) \in$ $C h_{c}\left(\tilde{X}^{\prime} \cup\left\{\left(s, c, t^{\prime}, 2\right)\right\}\right)$ holds. Thus, $\tilde{X}^{\prime}$ is not HM-stable. 
Then assume $s$ claims an empty seat of $c$ by Condition (nw-iii) in Definition 9, i.e.,

$$
\begin{aligned}
& (s, c, t) \in X^{\prime},\left(s, c, t^{\prime}\right) \in X \backslash X^{\prime},\left(s, c, t^{\prime}\right) \succ_{s}(s, c, t),\left(s, c, t^{\prime}\right) \succ_{c}(s, c, t), \\
& \left|X_{c, t}^{\prime}\right|>q_{c, t}, \text { and }\left|X_{c, t^{\prime}}^{\prime}\right| \geq q_{c, t^{\prime}}
\end{aligned}
$$

hold. Then in the fine-grained model, there exists a contract $\left(s^{\prime}, c, t, 3\right) \in \tilde{X}^{\prime}$ such that $(s, c, t, 3) \tilde{\succ}_{c}\left(s^{\prime}, c, t, 3\right)$ or $s^{\prime}=s$ holds. Thus, $\left(s, c, t^{\prime}, 3\right) \tilde{\succ}_{c}\left(s^{\prime}, c, t, 3\right)$ holds. Also,

$$
(s, c, t, k) \in \tilde{X}^{\prime},\left(s, c, t^{\prime}, 3\right) \in \tilde{X} \backslash \tilde{X}^{\prime}, \text { and }\left(s, c, t^{\prime}, 3\right) \tilde{\succ}_{s}(s, c, t, k)
$$

hold for some $k$. It is clear that $\left(s, c, t^{\prime}, 3\right) \in C h_{s}\left(\tilde{X}^{\prime} \cup\left\{\left(s, c, t^{\prime}, 3\right)\right\}\right)$ holds. Also, when calculating $C h_{c}\left(\tilde{X}^{\prime} \cup\left\{\left(s, c, t^{\prime}, 3\right)\right\}\right),\left(s, c, t^{\prime}, 3\right)$ is examined before $\left(s^{\prime}, c, t, 3\right)$, and thus, $\left(s, c, t^{\prime}, 3\right) \in$ $C h_{c}\left(\tilde{X}^{\prime} \cup\left\{\left(s, c, t^{\prime}, 3\right)\right\}\right)$ holds. Thus $\tilde{X}^{\prime}$ is not HM-stable.

Next assume $s$ claims an empty seat of $c^{\prime}$ by type by Condition (nw-iv) in Definition 9;

$$
(s, c, t) \in X^{\prime},\left(s, c^{\prime}, t^{\prime}\right) \in X \backslash X^{\prime},\left(s, c^{\prime}, t^{\prime}\right) \succ_{s}(s, c, t) \text {, and }\left|X_{c^{\prime}, t^{\prime}}\right|<p_{c^{\prime}, t^{\prime}}
$$

hold. Then in the fine-grained model,

$$
(s, c, t, k) \in \tilde{X}^{\prime},\left(s, c^{\prime}, t^{\prime}, 1\right) \in \tilde{X} \backslash \tilde{X}^{\prime}, \operatorname{and}\left(s, c^{\prime}, t^{\prime}, 1\right) \tilde{\succ}_{s}(s, c, t, k)
$$

hold for some $k$. It is clear that $\left(s, c^{\prime}, t^{\prime}, 1\right) \in C h_{s}\left(\tilde{X}^{\prime} \cup\left\{\left(s, c^{\prime}, t^{\prime}, 1\right)\right\}\right)$ and $\left(s, c^{\prime}, t^{\prime}, 1\right) \in$ $C h_{c^{\prime}}\left(\tilde{X}^{\prime} \cup\left\{\left(s, c^{\prime}, t^{\prime}, 1\right)\right\}\right)$ hold. Thus $\tilde{X}^{\prime}$ is not HM-stable. i.e.,

Finally, assume $s$ claims an empty seat of $c^{\prime}$ by type by Condition (nw-v) in Definition 9 ,

$$
(s, c, t) \in X^{\prime},\left(s, c^{\prime}, t^{\prime}\right) \in X \backslash X^{\prime},\left(s, c^{\prime}, t^{\prime}\right) \succ_{s}(s, c, t), p_{c^{\prime}, t^{\prime}} \leq\left|X_{c^{\prime}, t^{\prime}}^{\prime}\right|<q_{c^{\prime}, t^{\prime}} \text {, and }
$$

there exists $t^{\prime \prime}$ such that $\left|X_{c^{\prime}, t^{\prime \prime}}^{\prime}\right|>q_{c^{\prime}, t^{\prime \prime}}$

hold. Then in the fine-grained model,

$$
\begin{aligned}
& (s, c, t, k) \in \tilde{X}^{\prime},\left(s^{\prime}, c^{\prime}, t^{\prime \prime}, 3\right) \in \tilde{X}^{\prime},\left(s, c^{\prime}, t^{\prime}, 2\right) \in \tilde{X} \backslash \tilde{X}^{\prime},\left(s, c^{\prime}, t^{\prime}, 2\right) \tilde{\succ}_{s}(s, c, t, k), \text { and } \\
& \left|\tilde{X}_{c^{\prime}, t^{\prime}, 2}^{\prime}\right|<q_{c^{\prime}, t^{\prime}}-p_{c^{\prime}, t^{\prime}}
\end{aligned}
$$

hold for some $k$ and $s^{\prime}$. It is clear that $\left(s, c^{\prime}, t^{\prime}, 2\right) \in C h_{s}\left(\tilde{X}^{\prime} \cup\left\{\left(s, c^{\prime}, t^{\prime}, 2\right)\right\}\right)$ and $\left(s, c^{\prime}, t^{\prime}, 2\right) \in$ $C h_{c^{\prime}}\left(\tilde{X}^{\prime} \cup\left\{\left(s, c^{\prime}, t^{\prime}, 2\right)\right\}\right)$ hold. Thus, $\tilde{X}^{\prime}$ is not HM-stable.

Next, let us show that fairness and nonwastefulness imply HM-stability. Assume matching $\tilde{X}^{\prime}$ is not HM-stable, i.e., there exists $(s, c, t, k) \in \tilde{X} \backslash \tilde{X}^{\prime}$ such that

$$
(s, c, t, k) \in C h_{s}\left(\tilde{X}^{\prime} \cup\{(s, c, t, k)\}\right) \text { and }(s, c, t, k) \in C h_{c}\left(\tilde{X}^{\prime} \cup\{(s, c, t, k)\}\right)
$$

hold. Assume $\left(s, c^{\prime}, t^{\prime}, k^{\prime}\right) \in \tilde{X}^{\prime}$. It is clear that $(s, c, t, k) \tilde{\succ}_{s}\left(s, c^{\prime}, t^{\prime}, k^{\prime}\right)$ holds since $(s, c, t, k) \in$ $C h_{s}\left(\tilde{X}^{\prime} \cup\{(s, c, t, k)\}\right)$. If $k=1$ and $\left|\tilde{X}_{c, t}^{\prime}\right|<p_{c, t}$ hold, then $s$ claims an empty seat of $c$ by type since Condition (nw-iv) in Definition 9 holds. Also, if $k=1$ and $\left|\tilde{X}_{c, t}^{\prime}\right| \geq p_{c, t}$ hold, then $s$ has a justified envy toward some student accepted in $c$ with type $t$ since Condition (fr-i) in Definition 8 holds.

Thus assume $k \geq 2$. Assume $C h_{c}\left(\tilde{X}^{\prime} \cup\{(s, c, t, k)\}\right)=C h_{c}\left(\tilde{X}^{\prime}\right) \cup\{(s, c, t, k)\}$, i.e., no contract is rejected as the consequence of accepting $(s, c, t, k)$. Then $\left|X_{c}^{\prime}\right|<q_{c}$ must hold 
and $X^{\prime}$ is wasteful from Condition (nw-i) in Definition 9. Thus assume $\left(s^{\prime}, c, t^{\prime \prime}, k^{\prime \prime}\right) \in$ $C h_{c}\left(\tilde{X}^{\prime}\right)$ and $\left(s^{\prime}, c, t^{\prime \prime}, k^{\prime \prime}\right) \notin C h_{c}\left(\tilde{X}^{\prime} \cup\{(s, c, t, k)\}\right)$ hold, i.e., $\left(s^{\prime}, c, t^{\prime \prime}, k^{\prime \prime}\right)$ is rejected as the consequence of accepting $(s, c, t, k)$. If is clear $k \leq k^{\prime \prime}$ holds. If $k=2$ and $k^{\prime \prime}=3$, then $s$ claims an empty seat of $c$ by type since Condition (nw-iv) or (nw-v) in Definition 9 holds. Either $k=k^{\prime \prime}=2$ or $k=k^{\prime \prime}=3$ holds.

Let us consider the case where $s=s^{\prime}$. Then $\left(s, c, t^{\prime \prime}, k\right)$ is rejected as the consequence of accepting $(s, c, t, k)$. If $k=2$, then $s$ claims an empty seat of $c$ in $X^{\prime}$ since Condition (nw-ii) holds. If $k=3$, then $s$ claims an empty seat of $c$ in $X^{\prime}$ since Condition (nw-ii) or (nw-iii) in Definition 9 holds.

Finally, consider the case where $s \neq s^{\prime}$. Then (i) $t=t^{\prime \prime}$, (ii) $t \neq t^{\prime \prime}$ and $k=k^{\prime \prime}=2$, or (iii) $t \neq t^{\prime \prime}$ and $k=k^{\prime \prime}=3$. In case (i), $s$ has justified envy toward $s^{\prime}$ since $(s, c, t) \succ_{c}\left(s^{\prime}, c, t\right)$, and Condition (fr-i) in Definition 3 holds. In case (ii), $s$ has justified envy toward $s^{\prime}$ since Condition (fr-ii) in Definition 3 holds. In case (iii), $s$ has justified envy toward $s^{\prime}$ since Condition (fr-ii) or (fr-iii) in Definition 3 holds.

Lemma 4. $C h_{C}$ satisfies the irrelevance of rejected contracts, the law of aggregate demand, and the substitutes condition.

Proof. To show that these three properties hold, it is sufficient to show that each individual choice function $C h_{c}$ satisfies the following condition.

For any $\tilde{X}^{\prime} \subset \tilde{X}$ and $x \in \tilde{X} \backslash \tilde{X}^{\prime}, C h_{c}\left(\tilde{X}^{\prime} \cup\{x\}\right)$ is equal to

(i) $C h_{c}\left(\tilde{X}^{\prime}\right)$,

(ii) $C h_{c}\left(\tilde{X}^{\prime}\right) \cup\{x\}$, or

(iii) $\left(C h_{c}\left(\tilde{X}^{\prime}\right) \backslash\left\{x^{\prime}\right\}\right) \cup\{x\}$, where $x^{\prime} \in C h_{c}\left(\tilde{X}^{\prime}\right)$.

In words, by adding $x$ to $\tilde{X}^{\prime}$, the selected contracts do not change, $x$ is selected while all previously selected contracts are also selected, or $x$ is selected while all previously selected contracts except $x^{\prime}$ are also selected. This is called the single-improvement property (Gul \& Stacchetti, 1999).

Let us consider the procedure for calculating $C h_{c}\left(\tilde{X}^{\prime} \cup\{x\}\right)$, From Definition 10, it is clear that the outcome of each contract that is examined before $x$ does not change. Also, if $x$ is not accepted, i.e., it is not added to $Y$, the outcome of each contract that is examined after $x$ does not change. Thus, in this case, Condition (i), i.e., $C h_{c}\left(\tilde{X}^{\prime} \cup\{x\}\right)=$ $C h_{c}\left(\tilde{X}^{\prime}\right)$ holds. Assume $x=(s, c, t, k)$ is accepted and added to $Y$. When examining the remaining contracts, the only difference between the case of calculating $C h_{c}\left(\tilde{X}^{\prime} \cup\{x\}\right)$ and $C h_{c}\left(\tilde{X}^{\prime}\right)$ is that $x$ is added to $Y$. If no contract is rejected, then Condition (ii), i.e., $C h_{c}\left(\tilde{X}^{\prime} \cup\{x\}\right)=C h_{c}\left(\tilde{X}^{\prime}\right) \cup\{x\}$ holds. Assume there exists a rejected contract. More specifically, let $x^{\prime}=\left(s^{\prime}, c, t^{\prime}, k^{\prime}\right) \in C h_{c}\left(\tilde{X}^{\prime}\right)$ be the first rejected contract that is examined after $x$. Then, the reason why $x^{\prime}$ is rejected is one of the following:

(a) $|Y|=q_{c}$ holds in Step 3 or Step 4 before selecting $x^{\prime}$,

(b) $t^{\prime}=t, k=k^{\prime}=1$, and $\left|Y_{c, t, 1}\right|=p_{c, t}$ holds in Step 2 before selecting $x^{\prime}$, or

(c) $t^{\prime}=t, k=k^{\prime}=2$, and $\left|Y_{c, t, 2}\right|=q_{c, t}-p_{c, t}$ holds in Step 3 before selecting $x^{\prime}$. 
When (a) occurs, then $x^{\prime}$ is the last contract accepted in $C h_{c}\left(\tilde{X}^{\prime}\right)$. Thus, the outcome of each contract that is examined after $x^{\prime}$ does not change, i.e., it is rejected. When (b) occurs, then $x^{\prime}$ is the last contract accepted in Step 2 for $t$ in $C h_{c}\left(\tilde{X}^{\prime}\right)$. Thus, the outcome of each contract that is examined after $x^{\prime}$ in Step 2 for $t$ does not change, i.e., it is rejected.

Also, since $x$ is accepted and $x^{\prime}$ is rejected, $|Y|,\left|Y_{c, t, 1}\right|$, and $\left|Y_{c, t, 2}\right|$ become identical to the case in calculating $C h_{c}\left(\tilde{X}^{\prime}\right)$. Thus the outcome of each contract that is examined in Step 2 for other types, Steps 3 and 4, does not change. When (c) occurs, $x^{\prime}$ is the last contract accepted in Step 3 for $t$ in $C h_{c}\left(\tilde{X}^{\prime}\right)$. Thus the outcome of each contract that is examined after $x^{\prime}$ in Step 3 for $t$ does not change, i.e., it is rejected. Also, since $x$ is accepted and $x^{\prime}$ is rejected, $|Y|$ and $\left|Y_{c, t, 2}\right|$ become identical to the case in calculating $C h_{c}\left(\tilde{X}^{\prime}\right)$. Thus, the outcome of each contract that is examined in Step 3 for other types and Step 4, does not change. Thus if there exists rejected contract $x^{\prime}$, Condition (iii), i.e., $C h_{c}\left(\tilde{X}^{\prime} \cup\{x\}\right)=\left(C h_{c}\left(\tilde{X}^{\prime}\right) \backslash\left\{x^{\prime}\right\}\right) \cup\{x\}$ holds.

\section{References}

Abdulkadiroğlu, A. (2005). College admissions with affirmative action. International Journal of Game Theory, 33, 535-549.

Abdulkadiroğlu, A., Pathak, P. A., \& Roth, A. E. (2009). Strategy-proofness versus Efficiency in Matching with Indifferences: Redesigning the NYC High School Match. American Economic Review, 99, 1954-1978.

Abdulkadiroğlu, A., \& Sönmez, T. (2003). School choice: A mechanism design approach. American Economic Review, 93(3), 729-747.

Awasthi, P., \& Sandholm, T. (2009). Online stochastic optimization in the large: Application to kidney exchange. In Twenty-first International Joint Conference on Artifical Intelligence (IJCAI), pp. 405-411.

Biró, P., Fleiner, T., Irving, R., \& Manlove, D. (2010). The college admissions problem with lower and common quotas. Theoretical Computer Science, 411(34-36), 3136-3153.

Braun, S., Dwenger, N., Kübler, D., \& Westkamp, A. (2014). Implementing quotas in university admissions: An experimental analysis. Games and Economic Behavior, 85, $232-251$.

Budish, E., Che, Y.-K., Kojima, F., \& Milgrom, P. R. (2010). Designing random allocation mechanisms: Theory and applications. American Economic Review, 103, 585-623.

Drummond, J., \& Boutilier, C. (2013a). Elicitation and approximately stable matching with partial preferences. In Twenty-third International Joint Conference on Artificial Intelligence (IJCAI), pp. 97-105.

Drummond, J., \& Boutilier, C. (2013b). Elicitation and approximately stable matching with partial preferences. In Proceedings of the Twenty-Third International Joint Conference on Artificial Intelligence, pp. 97-105.

Dur, U., Kominers, S. D., Pathak, P. A., \& Sönmez, T. (2013). The demise of walk zones in Boston: Priorities vs. precedence in school choice.. working paper, Boston College. 
Echenique, F., \& Yenmez, M. B. (2015). How to control controlled school choice. American Economic Review, 105(8), 2679-2694.

Ehlers, L., Hafalir, I. E., Yenmez, M. B., \& Yildirim, M. A. (2014). School choice with controlled choice constraints: Hard bounds versus soft bounds. Journal of Economic Theory, 153, 648-683.

Ergin, H., \& Sönmez, T. (2006). Games of school choice under the Boston mechanism. Journal of Public Economics, 90, 215-237.

Fleiner, T. (2003). A fixed-point approach to stable matchings and some applications. Mathematics of Operations Research, 28, 103-126.

Fleiner, T., \& Kamiyama, N. (2016). A matroid approach to stable matchings with lower quotas. Mathematics of Operations Research. Forthcoming.

Fragiadakis, D., Iwasaki, A., Troyan, P., Ueda, S., \& Yokoo, M. (2016). Strategyproof matching with minimum quotas. ACM Transactions on Economics and Computation, $4(1), 6: 1-6: 40$.

Goto, M., Hashimoto, N., Iwasaki, A., Kawasaki, Y., Ueda, S., Yasuda, Y., \& Yokoo, M. (2014). Strategy-proof matching with regional minimum quotas. In Thirteenth International Conference on Autonomous Agents and Multiagent Systems (AAMAS), pp. $1225-1232$.

Goto, M., Iwasaki, A., Kawasaki, Y., Kurata, R., Yasuda, Y., \& Yokoo, M. (2016). Strategyproof matching with regional minimum and maximum quotas. Artificial Intelligence, 235, 40 - 57.

Goto, M., Iwasaki, A., Kawasaki, Y., Yasuda, Y., \& Yokoo, M. (2014). Improving fairness and efficiency in matching markets with regional caps: Priority-list based deferred acceptance mechanism. Mimeo (the latest version is available at http://mpra.ub.unimuenchen.de/53409/).

Goto, M., Kojima, F., Kurata, R., Tamura, A., \& Yokoo, M. (2016). Designing matching mechanisms under general distributional constraints. American Economic Review: Microeconomics, Forthcoming. (a draft version is available at http://mpra.ub.unimuenchen.de/64000/).

Gul, F., \& Stacchetti, E. (1999). Walrasian equilibrium with gross substitutes. Journal of Economic Theory, 87(1), 95-124.

Hafalir, I. E., Yenmez, M. B., \& Yildirim, M. A. (2013). Effective affirmative action in school choice. Theoretical Economics, 8(2), 325-363.

Hamada, K., Iwama, K., \& Miyazaki, S. (2016). The hospitals/residents problem with lower quotas. Algorithmica, $74(1), 440-465$.

Hatfield, J. W., \& Milgrom, P. R. (2005). Matching with contracts. American Economic Review, 95(4), 913-935.

Hatfield, J. W., \& Kojima, F. (2008). Matching with contracts: Comment. American Economic Review, 98(3), 1189-94.

Hatfield, J. W., \& Kojima, F. (2009). Group incentive compatibility for matching with contracts. Games and Economic Behavior, 67(2), $745-749$. 
Huang, C.-C. (2010). Classified stable matching. In Proceedings of ACM-SIAM Symposium on Discrete Algorithms (SODA-2010), pp. 1235-1253.

Kamada, Y., \& Kojima, F. (2014). General theory of matching under distributional constraints. Mimeo.

Kamada, Y., \& Kojima, F. (2015). Efficient matching under distributional constraints: Theory and applications. American Economic Review, 105(1), 67-99.

Kamiyama, N. (2013). A note on the serial dictatorship with project closures. Operations Research Letters, 41, 559-561.

Kojima, F. (2012). School choice: Impossibilities for affirmative action. Games and Economic Behavior, 75(2), 685-693.

Kojima, F., Tamura, A., \& Yokoo, M. (2014). Designing matching mechanisms under constraints: An approach from discrete convex analysis. In Proceedings of the Seventh International Symposium on Algorithmic Game Theory (SAGT-2014) (the full version is available at http://mpra.ub.uni-muenchen.de/56189).

Kominers, S. D., \& Sönmez, T. (2015). Matching with slot-specific priorities: Theory. Theoretical Economics. Forthcoming.

Kurata, R., Goto, M., Iwasaki, A., \& Yokoo, M. (2015). Controlled school choice with soft bounds and overlapping types. In Proceedings of the Twenty-Ninth AAAI Conference on Artificial Intelligence, pp. 951-957.

Lu, T., \& Boutilier, C. (2014). Effective sampling and learning for Mallows models with pairwise-preference data. Journal of Machine Learning Research, 15, 3963-4009.

Manlove, D. (2013). Algorithmics of Matching Under Preferences. World Scientific Publishing Company.

Monte, D., \& Tumennasan, N. (2013). Matching with quorum. Economic Letters, 120, $14-17$.

Perrault, A., Drummond, J., \& Bacchus, F. (2016). Strategy-proofness in the stable matching problem with couples. In Proceedings of the 2016 International Conference on Autonomous Agents and Multiagent Systems, pp. 132-140.

Pini, M. S., Rossi, F., \& Venable, K. B. (2014). Stable matching problems with soft constraints. In Thirteenth International Conference on Autonomous Agents and Multiagent Systems (AAMAS), pp. 1511-1512.

Rastegari, B., Condon, A., Immorlica, N., \& Leyton-Brown, K. (2013). Two-sided matching with partial information. In Proceedings of the Fourteenth ACM Conference on Electronic Commerce, pp. 733-750.

Roth, A. E., \& Sotomayor, M. A. O. (1990). Two-Sided Matching: A Study in GameTheoretic Modeling and Analysis (Econometric Society Monographs). Cambridge University Press.

Sönmez, T., \& Switzer, T. B. (2013). Matching with (branch-of-choice) contracts at the United States Military Academy. Econometrica, 81(2), 451-488.

Tubbs, J. D. (1992). Distance Based Binary Matching, pp. 548-550. Springer New York. 
Kurata, Hamada, Iwasaki, and Yokoo

Westkamp, A. (2013). An analysis of the German university admission system. Economic Theory, 53, 561-589.

Yokoi, Y. (2014). Matroidal choice functions. Mathematical Engineering Technical Reports, 2014-32, 1-27. 